MATHEMATICS OF COMPUTATION

Volume 73, Number 245, Pages 143-167

S 0025-5718(03)01581-3

Article electronically published on July 1, 2003

\title{
DISCRETE FREDHOLM PROPERTIES AND CONVERGENCE ESTIMATES FOR THE ELECTRIC FIELD INTEGRAL EQUATION
}

\author{
SNORRE H. CHRISTIANSEN
}

\begin{abstract}
The Galerkin discretization of the Electric Field Integral Equation is reinvestigated. We prove quasi-optimal convergence estimates at nonresonant frequencies, using orthogonal splittings of the Galerkin space. At resonant frequencies we show that the spurious electric currents radiate only weakly in the exterior domain. This is achieved through the study of some finitely degenerated problems in terms of LBB Inf-Sup estimates and the use of discrete Helmholtz decompositions.
\end{abstract}

\section{INTRODUCTION}

The Electric Field Integral Equation (EFIE) is defined in Section [2.1, It is the basic equation in the theory of integral methods for the scattering of electromagnetic harmonic (i.e., mono-frequential) waves. The discretization of this equation is a widespread method for the computation of radar cross sections, antenna performance and electromagnetic compatibility issues, to mention just a few important fields of application.

At nonresonant frequencies we give a new and rather general proof of the Inf-Sup condition for the Galerkin discretization of the EFIE on a large class of Galerkin spaces. The challenge is that the operator is not a compact perturbation of a coercive form. To compensate for this, we use splittings of the Galerkin spaces into orthogonal sums, where one of the two subspaces is the kernel of the divergence operator. We prove that the discrete Inf-Sup condition holds whenever the gap from the other subspace to its continuous analogue (the orthogonal of the kernel of the divergence operator) tends to zero. In turn this condition is shown to hold whenever discrete Helmholtz decompositions of tangent fields (analogues of the decompositions $u=\operatorname{grad} p+v$ with $\operatorname{div} v=0$ ) are sufficiently well behaved, which is checked for the standard spaces. We deduce quasi-optimal convergence rates in natural norms, for the approximate solutions in standard surface Finite Element spaces.

At resonant frequencies the integral operator appearing in the EFIE is degenerate. However the operator is of Fredholm type, and for exterior problems the righthand side is in general compatible - that is, the EFIE has a solution. We address the question as to what happens for the discretized equation. When the right-hand

Received by the editor December 26, 2000 and, in revised form, April 10, 2002.

2000 Mathematics Subject Classification. Primary 65N12, 65N38, 78M15.

This work received financial support from Thales Airborne Systems.

(C)2003 American Mathematical Society 
side is compatible, it is a straightforward remark that there exist bounded families (indexed by the mesh refinement parameter $h$ ) of approximate solutions to the discrete equations, for instance the best approximations in any chosen norm. We prove a converse property, namely that for any bounded family of approximate solutions the exterior electromagnetic field is well approximated. Since by the above remark it suffices to consider only the homogeneous equation, an interpretation of this result is that discrete spurious currents radiate much less in the exterior domain than their norm would indicate: they are close to the kernel of the continuous EFIE operator.

Other integral equations are currently used to solve electromagnetic scattering problems. Some of these, such as the Magnetic Field Integral Equation (MFIE) are also degenerate only for a discrete set of frequencies. However at these, the righthand side is usually not compatible. Linear combinations of the EFIE and MFIE known as CFIEs avoid the existence of resonances at the expense of introducing a somewhat arbitrary (complex) parameter. This is also known as the Brakhage and Werner trick. The EFIE has the advantage of having an unknown with a physical interpretation: it is the electric current density on the surface and has been perceived to be more robust on singular scatterers; in particular CFIEs cannot treat open surfaces (screens). However a more elaborate comparison is beyond the goals of this paper.

One of the main motivations for constructing the present theory was to provide a rigorous justification for the use of a new nonlocal finite element space, which appeared naturally in a preconditioning technique for the EFIE described in Christiansen-Nédélec [16]. We therefore remain sufficiently general throughout the paper to provide a theory that applies to such nonstandard spaces.

To achieve our goals the three main tools used are

- a reformulation of the EFIE as an equivalent saddle-point problem, following a technique used by Nédélec [29] to prove the Fredholm property of the (continuous) EFIE. It enables us to argue in terms of compact perturbations of coercive forms.

- estimates on discrete Helmholtz decompositions closely related to a discrete compactness result due to Kikuchi [24]. They have recently been found to be important in the discretization of eigenvalue problems by saddle-point formulations.

- a general theory of the discretization of finitely degenerated problems, which we have tried to develop in the spirit of Babuska's Inf-Sup conditions [2].

The most well-known Galerkin space is the one introduced for the EFIE by RaoWilton-Glisson in [32]. This space of tangent fields on $\Gamma$ had previously been used by Nédélec [27] for the computation of eddy currents and, as noted by him, is an adaptation to arbitrary surfaces of the lowest degree Raviart-Thomas (RT) finite element (FE) space developed for planar problems [33. A numerical analysis of the Galerkin discretization of the EFIE by RT FE of any order was first presented by Bendali [5]. We improve the results of Bendali by getting rid of a parasitic factor of the form $C h^{-1 / 2-\epsilon}$ (for arbitrary $\epsilon>0, C$ depending on $\epsilon$ ) in the error estimates. It should be remarked that we do not need to compute any residual error in the sense of Hsiao-Kleinman [21] to assess the quality of the solution. 
The paper is organized as follows:

Section 1: We develop a theory of discrete Fredholm properties in an abstract setting.

Section 2, We recall the main results we need on the integral representations of electromagnetic fields and the related Electric Field Integral Equation. In particular we introduce the appropriate splitting of the solution space.

Section 3: We turn to the discretization and prove the Inf-Sup condition for the EFIE and the other announced results using the theory of the first two sections.

\section{Discrete Fredholm Properties}

The EFIE is an example of an equation $A u=v$ involving a Fredholm operator $A$ and a right-hand side $v$ which in many cases of practical interest is compatible. In this section we develop a tool for studying the discretization of Fredholm operators by Galerkin methods. First we recall the definition of a left semi-Fredholm (LSF) operator and Babuska's Inf-Sup conditions for the solvability of equations involving invertible operators. Then we propose a definition for a discrete LSF condition. It is a generalisation of Babuska's Inf-Sup condition such that

- if an injective operator is discrete LSF (on a given family of Galerkin spaces), then it satisfies a standard (one-sided) Babuska Inf-Sup condition (Corollary 1.11);

- if an operator is discrete LSF, then so is any compact perturbation of it (Corollary 1.13).

The combination of these two properties is practical for proving Inf-Sup conditions for injective operators since, in the course of such a proof, many compact perturbations are sometimes involved, but injectivity is not guaranteed in the intermediate steps. In this paper, one compact perturbation appears once appropriate splittings are considered for the EFIE (Theorem 2.6); others are due to the relative compacity of the frequency part $G_{k}-G_{0}$ of the Green kernel.

1.1. Results on left semi-Fredholm operators. We collect some well-known facts that will be useful later and that will serve as references for discrete counterparts. The classical reference on the subject is Kato 23 .

Theorem 1.1. Let $X$ and $Y$ be two Banach spaces and $A: X \rightarrow Y$ be linear and continuous. The following conditions are equivalent:

(1) A has closed range and finite dimensional kernel;

(2) $\operatorname{dim} \operatorname{ker} A<\infty$ and on any closed supplementary $M$ of $\operatorname{ker} A$

$$
\inf _{u \in M}\|A u\| /\|u\|>0
$$

(3) there is a closed subspace $M$ of $X$ with finite codimension such that

$$
\inf _{u \in M}\|A u\| /\|u\|>0 .
$$

Definition 1.2. Let $X$ and $Y$ be two Banach spaces and $A: X \rightarrow Y$ be linear and continuous. We say that $A$ is left semi-Fredholm (LSF) if the stated conditions are satisfied. The set of LSF operators from $X$ to $Y$ is denoted $\operatorname{LSF}(X, Y)$. 
Theorem 1.3. Let $X$ and $Y$ be two Banach spaces. For all $n \in \mathbb{N}$ the following sets (whose union is $\operatorname{LSF}(X, Y)$ ) are open in $\mathrm{L}(X, Y)$ :

$$
\{A \in \mathrm{L}(X, Y): A \text { has closed range and } \operatorname{dim} \operatorname{ker} A \leq n\} .
$$

Moreover $\operatorname{LSF}(X, Y)$ is stable under translation by compact operators.

1.2. Galerkin methods in the presence of Gårding inequalities. Let $X$ be a Hilbert space. The base field $\mathbb{K}$ of $X$ is either $\mathbb{R}$ or $\mathbb{C}$. Let $a: X \times X \rightarrow \mathbb{K}$ be a $\mathbb{K}$ bilinear continuous form on $X$. We say that $a$ is nondegenerate if the induced map $\mathcal{A}: X \rightarrow X^{\star}, u \mapsto a(u, \cdot)$ is an isomorphism (since $X$ is reflexive, this is equivalent to $u \mapsto a(\cdot, u)$ being one). When $a$ is nondegenerate, we construct approximations of $\mathcal{A}^{-1}$ by choosing a family of closed subspaces $\left(X_{h}\right)$ of $X$, which is approximating in the sense that

$$
\forall u \in X \quad \lim _{h} \inf _{u^{\prime} \in X_{h}}\left\|u-u^{\prime}\right\|=0 .
$$

Then for a given $l \in X^{\star}$ we seek solutions of

$$
u \in X_{h} \quad \forall u^{\prime} \in X_{h} \quad a\left(u, u^{\prime}\right)=l\left(u^{\prime}\right) .
$$

In accordance with usual conventions it is implicit that $\left(X_{h}\right)=\left(X_{h}\right)_{h \in H}$, where $H$ is some subset of $\mathbb{R}_{+}^{*}$ accumulating at 0 , and that $\lim _{h}$ means $\lim _{h \rightarrow 0}$.

Let $\mathcal{A}_{h}$ be the map: $X_{h} \rightarrow X_{h}^{\star}, u \mapsto a(u, \cdot)$. Then equation (1.5) can be restated as $u \in X_{h} \quad \mathcal{A}_{h} u=\left.l\right|_{X_{h}}$. If $\mathcal{A}_{h}$ is invertible and if

$$
\exists C>0 \forall l \in X^{\star} \forall h \quad\left\|\mathcal{A}^{-1} l-\left.\mathcal{A}_{h}^{-1} l\right|_{X_{h}}\right\| \leq C \inf _{u^{\prime} \in X_{h}}\left\|\mathcal{A}^{-1} l-u^{\prime}\right\|,
$$

we say that the Galerkin method yields quasi-optimal convergence.

A sufficient condition for $a$ to be nondegenerate is that it is coercive in the sense that for some $\mathbb{R}$-linear isometric involution of $X$, denoted $u \mapsto \bar{u}$ and called conjugation, we have

$$
\exists C>0 \forall u \in X \quad \mathfrak{R e} a(u, \bar{u}) \geq(1 / C)\|u\|^{2} .
$$

This is the Lax-Milgram Theorem. If $a$ is coercive, Céa's Lemma asserts that if the spaces $X_{h}$ are stable under conjugation (i.e., $u \mapsto \bar{u}$ maps $X_{h}$ into $X_{h}$ ), the Galerkin method yields quasi-optimal convergence.

In the context of boundary integral equations one soon encounters bilinear forms satisfying only a weaker estimate, known as a Gårding inequality:

$$
\exists C>0 \forall u \in X \quad \mathfrak{R e} a(u, \bar{u}) \geq(1 / C)\|u\|_{X}^{2}-C\|u\|_{Y}^{2},
$$

where $Y$ is a Hilbert space containing $X$ and such that the canonical injection $X \rightarrow Y$ is compact.

It was soon recognized that if $a$ is nondegenerate and satisfies a Gårding inequality, and if the spaces $X_{h}$ are stable under conjugation, then there exists $h_{0}>0$ such that the Galerkin method has quasi-optimal convergence on $\left(X_{h}\right)_{h<h_{0}}$. Early contributions in this direction include Schatz [36]. This fact is naturally stated in terms of the Inf-Sup conditions due to Babuska [2], 3]. For an insightful account on this we refer to Demkowicz [19], who attributes the result to Mikhlin; see also Wendland [40]. For future reference we recall Babuska's Theorem: 
Theorem 1.4. Let $X$ and $Y$ be two reflexive Banach spaces. Let a be a continuous bilinear form on $X \times Y$. If

$$
\begin{aligned}
\inf _{u \in X} \sup _{v \in Y} \frac{|a(u, v)|}{\|u\|\|v\|} \geq \alpha & >0, \\
\forall v \in Y \quad(\forall u \in X a(u, v)=0) & \Rightarrow(v=0),
\end{aligned}
$$

then

$$
\inf _{v \in Y} \sup _{u \in X} \frac{|a(u, v)|}{\|v\|\|u\|}=\inf _{u \in X} \sup _{v \in Y} \frac{|a(u, v)|}{\|u\|\|v\|},
$$

and for all $l \in Y^{\star}$ there is a unique $u \in X$ such that

$$
\forall v \in Y \quad a(u, v)=l(v) .
$$

It satisfies $\|u\| \leq \alpha^{-1}\|l\|$.

We will use the following notation whenever it makes sense:

$$
\sup _{Y} \mathcal{A}=\sup _{v \in Y} \frac{\|\mathcal{A} v\|}{\|v\|}, \quad \inf _{X} \sup _{Y} \mathcal{A}=\inf _{X} \sup _{Y} a=\inf _{u \in X} \sup _{v \in Y} \frac{|a(u, v)|}{\|u\|\|v\|}
$$

where it is implicit that $u$ and $v$ are nonzero.

1.3. Discrete left semi-Fredholm operators. The following lemma is trivial and stated just for the record.

Lemma 1.5. Let $X$ and $Y$ be two Banach spaces, and let $\mathcal{A}: X \rightarrow Y^{\star}$ be continuous. Let $M$ be a closed subspace of $X$. Let $\left(X_{h}\right)$ and $\left(Y_{h}\right)$ be families of closed subspaces of $X$ and $Y$. Suppose $\left(X_{h}\right)$ is approximating (and $\left.Y_{h} \neq\{0\}\right)$. If

$$
\liminf _{h} \inf _{X_{h} \cap M} \sup _{Y_{h}} \mathcal{A}>0,
$$

then $M \cap \operatorname{ker} \mathcal{A}=\{0\}$. If in addition $M$ has finite codimension, then $\mathcal{A}$ is LSF.

We will need two more lemmas, which are less trivial.

Lemma 1.6. Let $X$ be a Banach space. Let $M$ and $N$ be closed subspaces of $X$ such that $M \oplus N=X$ and $N$ is finite dimensional. Let $P: X \rightarrow X$ be the projector with range $M$ and kernel $N$. Let $\left(X_{h}\right)$ be a family of closed subspaces of $X$ which is approximating. Then

- there are projectors $P_{h}$ with range $M$ which converge in norm to $P$ and which leave $X_{h}$ stable for sufficiently small $h$;

- if $\left(P_{h}\right)$ is any family of such projectors, then for sufficiently small $h$, $\operatorname{ker} P_{h} \subset X_{h}$ and $X_{h}=\left(X_{h} \cap M\right) \oplus \operatorname{ker} P_{h}$.

Proof. Choose a basis $\left(e^{i}\right)$ of $N$, and pick elements $e_{h}^{i}$ of $X_{h}$ such that

$$
\lim _{h}\left\|e^{i}-e_{h}^{i}\right\|=0 .
$$

For any $h$, let $N_{h}$ be the space spanned by the $e_{h}^{i}$. Then there is $h_{0}>0$ such that for $h<h_{0}$ we have $X=M \oplus N_{h}$.

(1) For $h<h_{0}$ let $P_{h}$ be the projector with range $M$ and kernel $N_{h}$, and for $h \geq h_{0}$ put $P_{h}=P$. One checks that the family $\left(P_{h}\right)$ has the desired properties.

(2) If $\left(P_{h}\right)$ is a family of projectors with these properties, then $\left.P_{h}\right|_{X_{h}}$ are projectors with range $X_{h} \cap M$, so for sufficiently small $h$

$$
\operatorname{dim} N_{h} \leq\left.\operatorname{dim} \operatorname{ker} P_{h}\right|_{X_{h}} \leq \operatorname{dim} \operatorname{ker} P_{h} \leq \operatorname{dim} N .
$$

For sufficiently small $h$ these dimensions are all equal. 
Lemma 1.7. Let $X$ be a Banach space and $Y$ be a reflexive Banach space. Let $\mathcal{A}: X \rightarrow Y^{\star}$ be continuous and let it have closed range. Then for any $l \in X^{\star}$ that vanishes on $\operatorname{ker} \mathcal{A}$ there is a $v \in Y$ such that

$$
\forall u \in X \quad l(u)=(\mathcal{A} u)(v) .
$$

Proof. Remark first that $\mathcal{A}$ induces an isomorphism of Banach spaces $X / \operatorname{ker} \mathcal{A} \rightarrow$ $\mathcal{A}(X)$. Let $\mathcal{Z}$ be the inverse mapping. For convenience we put $N=\operatorname{ker} \mathcal{A}$.

Pick $l \in X^{\star}$ that vanishes on $N$. Let $\tilde{l}$ be the canonical image of $l$ in $(X / N)^{\star}$. Put $f=\mathcal{Z}^{\star} \tilde{l} \in(\mathcal{A}(X))^{\star}$. Extend $f$ to a continuous linear form on $Y^{\star}$, and put $v=\left(i_{Y}\right)^{-1} f$. For all $u \in X$ we have

$$
(\mathcal{A} u)(v)=f(\mathcal{A} u)=\tilde{l}(\mathcal{Z} \mathcal{A} u)=\tilde{l}(\tilde{u}),
$$

where $\tilde{u}$ is the canonical image of $u$ in $X / N$. The proof is complete.

Theorem 1.8. Let $X$ and $Y$ be two reflexive Banach spaces, and $\mathcal{A}: X \rightarrow Y^{\star}$ be LSF. Let $\left(X_{h}\right)$ and $\left(Y_{h}\right)$ be families of closed subspaces of $X$ and $Y$ which are approximating.

Put $N_{0}=\operatorname{ker} \mathcal{A}$. Suppose we have closed subspaces $M_{0}$ and $M_{1}$ such that $M_{0} \oplus$ $N_{0}=X, M_{1} \oplus N_{1}=M_{0}$ and $\operatorname{dim} N_{1}<\infty$. If

$$
\liminf _{h} \inf _{X_{h} \cap M_{1}} \sup _{Y_{h}} \mathcal{A}>0
$$

then

$$
\liminf _{h} \inf _{X_{h} \cap M_{0}} \sup _{Y_{h}} \mathcal{A}>0 .
$$

Proof. If not, let $\left(u_{n}\right),\left(h_{n}\right)$ be sequences such that

$$
u_{n} \in X_{h_{n}} \cap M_{0}, \quad\left\|u_{n}\right\|=1, \quad \lim _{n} h_{n}=0, \quad \limsup _{n} \sup _{Y_{h_{n}}} \mathcal{A} u_{n}=0 .
$$

Let $P$ be the projector with range $M_{1}$ and kernel $N_{1} \oplus N_{0}$. The sequence $\left(u_{n}-P u_{n}\right)$ is a bounded sequence in the finite dimensional space $N_{1} \oplus N_{0}$, so modulo extraction we can suppose that it converges in norm to some $u^{N} \in N_{1} \oplus N_{0}$. Since $u_{n} \in M_{0}$, we actually have $u^{N} \in N_{1}$.

Since $\left(Y_{h_{n}}\right)$ is approximating, we have

$$
\forall v \in Y \quad \lim _{n}\left(\mathcal{A} u_{n}\right)(v)=0
$$

Therefore, by Lemma 1.7 for any $l \in X^{\star}$ that vanishes on $N_{0}$ we have

$$
\lim _{n} l\left(u_{n}\right)=0 .
$$

If $l \in X^{\star}$ vanishes on $M_{1} \oplus N_{0}$, we have

$$
l\left(u^{N}\right)=\lim _{n} l\left(u_{n}-P u_{n}\right),
$$

and $P u_{n} \in M_{1}$ so this limit must be 0 . Therefore, by the Hahn-Banach theorems, $u^{N} \in M_{1} \oplus N_{0}$, so $u^{N}=0$.

Let $P_{h}: X \rightarrow X$ be projectors with range $M_{1}$, leaving $X_{h}$ stable and converging in norm to $P$, as in Lemma 1.6 Notice that

$$
\lim _{h}\left\|u_{n}-P_{h_{n}} u_{n}\right\|=0 .
$$

One obtains a contradiction using the discrete Inf-Sup estimate on $\left(X_{h} \cap M_{1}\right) \times Y_{h}$ for the sequence $\left(P_{h_{n}} u_{n}\right)$. 
Theorem 1.9. Let $X$ and $Y$ be two reflexive Banach spaces and $\mathcal{A}: X \rightarrow Y^{\star}$ be LSF. Let $\left(X_{h}\right)$ and $\left(Y_{h}\right)$ be families of closed subspaces of $X$ and $Y$ which are approximating.

Let $N$ denote the kernel of $\mathcal{A}$ and let $M_{1}$ and $M_{2}$ be two supplementaries of $N$ in $X$. If

$$
\liminf _{h} \inf _{X_{h} \cap M_{1}} \sup _{Y_{h}} \mathcal{A}>0
$$

then

$$
\liminf _{h} \inf _{X_{h} \cap M_{2}} \sup _{Y_{h}} \mathcal{A}>0 .
$$

Proof. Choose $\alpha>0$ and $h_{0}$ such that for all $h<h_{0}$

$$
\inf _{X_{h} \cap M_{1}} \sup _{Y_{h}} \mathcal{A} \geq \alpha .
$$

Let $P$ denote the projection with range $M_{1}$ and kernel $N$. Since $P$ induces a continuous bijection $M_{2} \rightarrow M_{1}$, by the standard theorems there is $\beta>0$ such that

$$
\forall u \in M_{2} \quad\|P u\| \geq \beta\|u\| .
$$

Moreover let $P_{h}: X \rightarrow X$ be projectors with range $M_{1}$, leaving $X_{h}$ stable and converging in norm to $P$, as in Lemma 1.6 .

For all $u \in X_{h} \cap M_{2}$ and all $v \in Y_{h}$ we have

$$
\begin{aligned}
|(\mathcal{A} u)(v)| & =|(\mathcal{A} P u)(v)| \\
& \geq\left|\left(\mathcal{A} P_{h} u\right)(v)\right|-\left|\left(\mathcal{A}\left(P-P_{h}\right) u\right)(v)\right| .
\end{aligned}
$$

Hence for $h<h_{0}$

$$
\sup _{Y_{h}} \mathcal{A} u \geq \alpha\left\|P_{h} u\right\|-\|\mathcal{A}\|\left\|P-P_{h}\right\|\|u\|
$$

Moreover

$$
\left\|P_{h} u\right\| \geq\|P u\|-\left\|\left(P-P_{h}\right) u\right\| \geq \beta\|u\|-\left\|P-P_{h}\right\|\|u\|,
$$

SO

$$
\sup _{Y_{h}} \mathcal{A} u \geq \alpha \beta\|u\|-(\|\mathcal{A}\|+\alpha)\left\|P-P_{h}\right\|\|u\| .
$$

It follows that

$$
\inf _{X_{h} \cap M_{2}} \sup _{Y_{h}} \mathcal{A} \geq \alpha \beta-(\|\mathcal{A}\|+\alpha)\left\|P-P_{h}\right\| .
$$

Definition 1.10. Let $X$ and $Y$ be two reflexive Banach spaces, and let $\mathcal{A}: X \rightarrow Y^{\star}$ be continuous. Let $\left(X_{h}\right)$ and $\left(Y_{h}\right)$ be families of closed subspaces of $X$ and $Y$. We say that $\mathcal{A}$ is discrete $L S F$ on $\left(X_{h} \times Y_{h}\right)$ if there is a closed subspace $M$ of $X$, with finite codimension in $X$ such that

$$
\liminf _{h} \inf _{X_{h} \cap M} \sup _{Y_{h}} \mathcal{A}>0 .
$$

In this situation, if we wish to be more precise, we say that $\mathcal{A}$ is discrete LSF on $\left(X_{h} \times Y_{h}\right)$ with respect to (w.r.t.) $M$. 
Corollary 1.11. With the hypotheses of Theorem 1.8 , if $\mathcal{A}$ is discrete LSF on $\left(X_{h} \times Y_{h}\right)$ w.r.t. $M$, then it is so w.r.t. any closed subspace whose intersection with $\operatorname{ker} \mathcal{A}$ is $\{0\}$. In particular if in addition $\mathcal{A}$ is injective, then $\mathcal{A}$ satisfies a uniform discrete Inf-Sup condition on $\left(X_{h} \times Y_{h}\right)$ in the sense of Babuska for small enough parameters $h$.

\subsection{Compact perturbations of discrete LSF operators.}

Theorem 1.12. Let $X$ and $Y$ be two reflexive Banach spaces, and let $\mathcal{A}: X \rightarrow Y^{\star}$ be LSF. Let $\mathcal{B}: X \rightarrow Y^{\star}$ be compact. Let $\left(X_{h}\right)$ and $\left(Y_{h}\right)$ be families of closed subspaces of $X$ and $Y$. Suppose $\left(Y_{h}\right)$ is approximating.

Suppose furthermore that $M_{0}$ is a closed subspace of $X$ that satifies

$$
\liminf _{h} \inf _{X_{h} \cap M_{0}} \sup _{Y_{h}} \mathcal{A}>0 .
$$

Let $N_{1}$ be the kernel of $\mathcal{A}+\mathcal{B}$ restricted to $M_{0}$, and suppose it has a closed supplementary $M_{1}$ in $M_{0}$ (i.e., $M_{0}=M_{1} \oplus N_{1}$ ). Then

$$
\liminf _{h} \inf _{X_{h} \cap M_{1}} \sup _{Y_{h}} \mathcal{A}+\mathcal{B}>0 .
$$

Proof. If not, let $\left(u_{n}\right),\left(h_{n}\right)$ be sequences such that

$$
u_{n} \in X_{h_{n}} \cap M_{1}, \quad\left\|u_{n}\right\|=1, \quad \lim _{n} h_{n}=0, \quad \limsup _{n} \sup _{Y_{h_{n}}}(\mathcal{A}+\mathcal{B}) u_{n}=0 .
$$

Since $\left(Y_{h_{n}}\right)$ is approximating, we have

$$
\forall v \in Y \quad \lim _{n}\left((\mathcal{A}+\mathcal{B}) u_{n}\right)(v)=0 .
$$

Therefore, for any continuous linear form $l \in X^{\star}$ that vanishes on $\operatorname{ker}(\mathcal{A}+\mathcal{B})$ we have

$$
\lim _{n} l\left(u_{n}\right)=0 .
$$

Any continuous linear form on $M_{1}$ has a continuous extension to $X$ that vanishes on $\operatorname{ker}(\mathcal{A}+\mathcal{B})$; therefore $\left(u_{n}\right)$ converges weakly to 0 in $M_{1}$, hence also in $X$. Since $\mathcal{B}$ is compact, $\left(\mathcal{B} u_{n}\right)$ converges in norm to 0 . One obtains a contradiction using the discrete Inf-Sup estimate for $\mathcal{A}$ on $\left(\left(X_{h} \cap M_{0}\right) \times Y_{h}\right)$.

Corollary 1.13. With the hypotheses of the above Theorem 1.12, if $\mathcal{A}$ is discrete $L S F$ on $\left(X_{h} \times Y_{h}\right)$, then so is $\mathcal{A}+\mathcal{B}$.

Example 1.14. If $X$ is a Hilbert space equipped with a conjugation and $\mathcal{A}: X \rightarrow$ $X^{\star}$ is continuous and satisfies a Gårding inequality, then $\mathcal{A}$ is discrete LSF on any approximating family of conjugation-stable and closed subspaces.

1.5. Error control of discrete LSF operators. The following property of discrete LSF operators will be important to us:

Theorem 1.15. Let $X$ and $Y$ be two reflexive Banach spaces, and let $\mathcal{A}: X \rightarrow Y^{\star}$ be LSF. Let $\left(X_{h}\right)$ and $\left(Y_{h}\right)$ be approximating families of closed subspaces of $X$ and $Y$. If $\mathcal{A}$ is discrete LSF on $\left(X_{h} \times Y_{h}\right)$, then there is $C>0$, such that for all $\epsilon>0$ there is $h_{\epsilon}>0$ such that for all $h<h_{\epsilon}$ and all $u \in X_{h}$

$$
\sup _{Y} \mathcal{A} u \leq C \sup _{Y_{h}} \mathcal{A} u+\epsilon\|u\| \text {. }
$$


Proof. Let $N$ be the kernel of $\mathcal{A}$ on $X$ and let $M$ be a closed supplementary of $N$ in $X$ such that $\mathcal{A}$ is discrete LSF w.r.t. $M$. Let $P$ be the projector with range $M$ and kernel $N$, and let $P_{h}$ be projectors as in Lemma [1.6. Pick $\alpha>0$ and $h_{0}$ such that for all $h<h_{0}, X_{h}$ is stable under $P_{h}$ and

$$
\inf _{X_{h} \cap M} \sup _{Y_{h}} \mathcal{A} \geq \alpha .
$$

Note first that for all $u \in X$,

$$
\mathcal{A} u=\mathcal{A P u} .
$$

In particular,

$$
\sup _{Y} \mathcal{A} u=\sup _{Y} \mathcal{A} P u \leq\|\mathcal{A}\|\|P u\|
$$

Moreover,

$$
\|P u\| \leq\left\|P_{h} u\right\|+\left\|P-P_{h}\right\|\|u\|,
$$

and if $u \in X_{h}$ and $h<h_{0}$,

$$
\left\|P_{h} u\right\| \leq \alpha^{-1} \sup _{Y_{h}} \mathcal{A} P_{h} u
$$

and

$$
\sup _{Y_{h}} \mathcal{A} P_{h} u \leq \sup _{Y_{h}} \mathcal{A} u+\|\mathcal{A}\|\left\|P-P_{h}\right\|\|u\| .
$$

All in all, if $u \in X_{h}$ and $h<h_{0}$,

$$
\sup _{Y} \mathcal{A} u \leq \alpha^{-1}\|\mathcal{A}\| \sup _{Y_{h}} \mathcal{A} u+\|\mathcal{A}\|\left(1+\alpha^{-1}\|\mathcal{A}\|\right)\left\|P-P_{h}\right\|\|u\| .
$$

The theorem follows.

Remark 1.16. Equation (1.49) together with the construction of projectors $P_{h}$ shown in point (1) of the proof of Lemma 1.6 yield rather explicit bounds on the constant $C$ and the threshold $h_{\epsilon}$ appearing in the theorem.

In particular under the hypotheses of the theorem, $\mathcal{A}$ has the following property:

$$
\begin{gathered}
\forall \epsilon>0 \exists \delta>0 \exists h_{0}>0 \forall h<h_{0} \forall u \in X_{h} \\
\left(\sup _{Y_{h}} \mathcal{A} u \leq \delta\|u\|\right) \Rightarrow\left(\sup _{Y} \mathcal{A} u \leq \epsilon\|u\|\right) .
\end{gathered}
$$

In other words, in a sense, small discrete residual error indicates small continuous residual error. This property is easily extended to hold uniformly for some families of operators, as follows:

Corollary 1.17. Let $X$ and $Y$ be two reflexive Banach spaces, and let $\mathfrak{A}$ be a compact subset of $\operatorname{LSF}\left(X, Y^{\star}\right)$ (in the norm topology). Let $\left(X_{h}\right)$ and $\left(Y_{h}\right)$ be approximating families of closed subspaces of $X$ and $Y$. If all $\mathcal{A}$ in $\mathfrak{A}$ are discrete LSF on $\left(X_{h} \times Y_{h}\right)$, then

$$
\begin{gathered}
\forall \epsilon>0 \exists \delta>0 \exists h_{0}>0 \forall h<h_{0} \forall u \in X_{h} \forall \mathcal{A} \in \mathfrak{A} \\
\left(\sup _{Y_{h}} \mathcal{A} u \leq \delta\|u\|\right) \Rightarrow\left(\sup _{Y} \mathcal{A} u \leq \epsilon\|u\|\right) .
\end{gathered}
$$


Proof. Choose a $\epsilon>0$. For each $\mathcal{A} \in \mathfrak{A}$ choose a $\delta(\mathcal{A})$ and a $h_{0}(\mathcal{A})$ so that holds for $\mathcal{A}$ relative to $\epsilon / 2$.

The family of balls with center $\mathcal{A}$ and radius $\min \{\delta(\mathcal{A}), \epsilon\} / 2$, for $\mathcal{A} \in \mathfrak{A}$, covers $\mathfrak{A}$, so we can extract a finite subcover indexed by, say, $\mathcal{A}_{1}, \ldots, \mathcal{A}_{n}$.

Put $\delta=\min _{i} \delta\left(\mathcal{A}_{i}\right) / 2$ and $h_{0}=\min _{i} h_{0}\left(\mathcal{A}_{i}\right)$. Take $\mathcal{A} \in \mathfrak{A}$ and choose $i$ so that $\left\|\mathcal{A}-\mathcal{A}_{i}\right\|$ is less than both $\delta\left(\mathcal{A}_{i}\right) / 2$ and $\epsilon / 2$.

For any $h<h_{0}$ and any $u \in X_{h}$ we have: If $\sup _{Y_{h}} \mathcal{A} u \leq \delta\|u\|$, then $\sup _{Y_{h}} \mathcal{A} u \leq$ $\left(\delta\left(\mathcal{A}_{i}\right) / 2\right)\|u\|$, so $\sup _{Y_{h}} \mathcal{A}_{i} u \leq \delta\left(\mathcal{A}_{i}\right)\|u\|$. This implies $\sup _{Y} \mathcal{A}_{i} u \leq(\epsilon / 2)\|u\|$, and hence $\sup _{Y} \mathcal{A} u \leq \epsilon\|u\|$.

\subsection{Extensions to quasi-conforming Galerkin approximations.}

Definition 1.18. Let $X$ be a Banach space and $X_{0}$ a closed subspace. Let $\left(X_{h}\right)$ be a family of closed subspaces of $X$. We say that

(1) $\left(X_{h}\right)$ is approximating in $X_{0}$ if

$$
\forall u \in X_{0} \quad \lim _{h} \inf _{u^{\prime} \in X_{h}}\left\|u-u^{\prime}\right\|=0
$$

(2) $\left(X_{h}\right)$ is quasi-conforming in $X_{0}$ if

$$
\lim _{h} \sup _{u \in X_{h}} \inf _{u^{\prime} \in X_{0}}\left\|u-u^{\prime}\right\| /\|u\|=0 .
$$

The quantity

$$
\delta\left(X_{h}, X_{0}\right)=\sup _{u \in X_{h}} \inf _{u^{\prime} \in X_{0}}\left\|u-u^{\prime}\right\| /\|u\|
$$

is called the gap from $X_{h}$ to $X_{0}$ (see Kato [23]).

Suppose $X_{0}$ has a closed supplementary $X_{1}$ in $X$ (one says that $X_{0}$ splits), and let $P: X \rightarrow X$ be the projection with range $X_{0}$ and kernel $X_{1}$. For all $u \in X$ one has

$$
\forall u^{\prime} \in X_{0} \quad\|u-P u\|=\left\|\left(u-u^{\prime}\right)-\left(P u-u^{\prime}\right)\right\|=\left\|\left(u-u^{\prime}\right)-P\left(u-u^{\prime}\right)\right\|,
$$

hence

$$
|\|u\|-\|P u\|| \leq\|u-P u\| \leq(1+\|P\|)\|u\| \inf _{u^{\prime} \in X_{0}}\left\|u-u^{\prime}\right\| /\|u\| .
$$

In particular if $\left(X_{h}\right)$ is a family of closed subspaces which is quasi-conforming in $X_{0}$, then for sufficiently small $h$ the spaces $P X_{h}$ are closed in $X_{0}$ and $P$ induces isomorphisms $X_{h} \rightarrow P X_{h}$ which are arbitrarily close in norm to the identity mapping on $X_{h}$. Also if in addition $\left(X_{h}\right)$ is approximating in $X_{0}$, then so is $\left(P X_{h}\right)$. From this we deduce the following lemma.

Lemma 1.19. Let $X$ and $Y$ be Banach spaces and let $\mathcal{A}: X \rightarrow Y^{\star}$ be continuous. Let $X_{0}$ and $Y_{0}$ be closed subspaces of $X$ and $Y$ that split-yielding projectors $P_{X}$ in $X$ and $P_{Y}$ in $Y$ as above - and $\mathcal{A}_{0}: X_{0} \rightarrow Y_{0}^{\star}$ be the map induced by $\mathcal{A}$. Let $\left(X_{h}\right)$ and $\left(Y_{h}\right)$ be families of closed subspaces of $X$ and $Y$, which are quasi-conforming in $X_{0}$ and $Y_{0}$ (i.e., the corresponding gaps tend to 0 ). For any closed $M_{0}$ in $X_{0}$, one has

$$
\liminf _{h} \inf _{M_{0} \cap P_{X} X_{h}} \sup _{P_{Y} Y_{h}} \mathcal{A}_{0}=\liminf _{h} \inf _{\left(M_{0} \oplus \operatorname{ker} P_{X}\right) \cap X_{h}} \sup _{Y_{h}} \mathcal{A} .
$$


With this lemma most results from the preceding sections carry over to the quasi-conforming setting in some form or another. However - as will be shown in the next section - the lemma is sufficient for our needs, so we will not develop this possibility here.

\section{The Electric Field Integral Equation}

2.1. Integral representation of interior and exterior waves. Let $\Omega_{-}$be an open bounded subset of $\mathbb{R}^{3}$, and let $\Gamma$ be its boundary. We suppose that $\bar{\Omega}_{-}=$ $\Omega_{-} \cup \Gamma$ is a $C^{\infty}$ smooth submanifold with boundary, though this hypothesis could be relaxed to $C^{p}$ smoothness for some $p<\infty$ without much extra effort. More interesting would have been an extension of the theory to Lipschitz manifolds, but even though scalar equations are well understood (see Costabel [18]), the case of Maxwell's equations is still an active research direction (see Buffa et al. [13]) which we will not pursue here. We denote by $\Omega_{+}$the complement of $\Omega_{-} \cup \Gamma$ and by $n$ the outward pointing normal on $\Gamma$. We suppose throughout that $\Omega_{+}$is connected, which ensures uniqueness of solutions to exterior problems.

The free-space harmonic Maxwell equations for the electromagnetic field $(E, H)$ in an open region $\Omega \subset \mathbb{R}^{3}$ are

$$
\begin{aligned}
\operatorname{curl} E & =+i \omega \mu H, \\
\operatorname{curl} H & =-i \omega \epsilon E,
\end{aligned}
$$

where $\mu>0$ is the magnetic permeability and $\epsilon>0$ is the electric permittivity. If the pulsation $\omega$ is zero, one also has to add explicitly that the fields are divergence free. In the sequel these equations will be referred to as Maxwell's equations. It is convenient to introduce the wavenumber $k$ and the impedance $Z$ defined by

$$
\begin{aligned}
k & =\omega(\mu \epsilon)^{1 / 2}, \\
Z & =(\mu / \epsilon)^{1 / 2} .
\end{aligned}
$$

Then we have $+i \omega \mu=+i k Z$ and $-i \omega \epsilon=-i k / Z$.

We will consider here only nonhomogeneous boundary value problems for the free-space harmonic Maxwell equations, with real nonzero positive pulsations. Referring to Colton-Kress [17], Cessenat [14] and Nédélec [29] for proofs, we briefly state the main results which will be of interest to us.

For any open domain $\Omega$ in $\mathbb{R}^{3}$ we use the notation

$$
\mathrm{H}_{\text {curl }}^{0}(\Omega)=\left\{u \in \mathrm{L}_{\mathrm{T}}^{2}(\Omega): \operatorname{curl} u \in \mathrm{L}_{\mathrm{T}}^{2}(\Omega)\right\},
$$

where $\mathrm{L}_{\mathrm{T}}^{2}(\Omega)$ denotes the space of square summable tangential fields. On $\Gamma$ the usual Sobolev spaces of scalar and tangential fields of regularity order $s \in \mathbb{R}$ are denoted $\mathrm{H}^{s}(\Gamma)$ and $\mathrm{H}_{\mathrm{T}}^{s}(\Gamma)$, respectively, and the corresponding norms are both written

$$
u \mapsto|u|_{s} .
$$

On $\Gamma$ we denote by div the surface divergence and introduce the Hilbert spaces $\mathrm{H}_{\text {div }}^{s}(\Gamma)$ of tangent fields on $\Gamma$

$$
\mathrm{H}_{\text {div }}^{s}(\Gamma)=\left\{u \in \mathrm{H}_{\mathrm{T}}^{s}(\Gamma): \operatorname{div} u \in \mathrm{H}^{s}(\Gamma)\right\} .
$$

They are equipped with the norms

$$
u \mapsto\|u\|_{s}:\|u\|_{s}^{2}=|u|_{s}^{2}+|\operatorname{div} u|_{s}^{2} .
$$


The surface rotational and the spaces $\mathrm{H}_{\text {rot }}^{s}(\Gamma)$ are defined in a similar way but we do not introduce any notation for the corresponding norm. Notice that $u \mapsto u \times n$ induces isomorphisms $\mathrm{H}_{\text {rot }}^{s}(\Gamma) \rightarrow \mathrm{H}_{\text {div }}^{s}(\Gamma)$ and $\mathrm{H}_{\text {div }}^{s}(\Gamma) \rightarrow \mathrm{H}_{\text {rot }}^{s}(\Gamma)$.

Recall that we have well-defined continuous tangential trace operators $([29]$, Theorem 5.4.2 p. 209)

$$
\gamma_{\mathrm{T}}^{-}:\left\{\begin{aligned}
\mathrm{H}_{\mathrm{curl}}^{0}\left(\Omega_{-}\right) & \rightarrow \mathrm{H}_{\mathrm{rot}}^{-1 / 2}(\Gamma) \\
v & \mapsto v_{\mathrm{T}}=v-(v \cdot n) n,
\end{aligned}\right.
$$

and, for arbitrary large enough $R>0$ (with $B_{R}=\left\{x \in \mathbb{R}^{3}:|x|<R\right\}$ )

$$
\gamma_{\mathrm{T}}^{+}:\left\{\begin{aligned}
\mathrm{H}_{\mathrm{curl}}^{0}\left(\Omega_{+} \cap B_{R}\right) & \rightarrow \mathrm{H}_{\mathrm{rot}}^{-1 / 2}(\Gamma), \\
v & \mapsto v_{\mathrm{T}}=v-(v \cdot n) n .
\end{aligned}\right.
$$

For simplicity we denote by $\mathrm{H}_{\text {curl }}^{0}\left(\Omega_{+}\right)_{\text {loc }}$ the Fréchet space of vector fields in $\Omega_{+}$ whose restrictions are in $\mathrm{H}_{\text {curl }}^{0}\left(\Omega_{+} \cap B_{R}\right)$ for all $R>0$.

The Silver-Müller radiation condition at infinity for an electromagnetic field $(E, H) \in \mathrm{H}_{\text {curl }}^{0}\left(\Omega_{+}\right)_{\text {loc }}^{2}$ is

$$
\mu^{1 / 2} H \times x /|x|-\epsilon^{1 / 2} E=o(1 /|x|) .
$$

For exterior problems we have the existence and uniqueness result ([29], Theorem 5.4.6, p. 220):

Theorem 2.1. For all $k>0$, all $v \in \mathrm{H}_{\text {rot }}^{-1 / 2}(\Gamma)$ there is a unique $(E, H) \in$ $\mathrm{H}_{\text {curl }}^{0}\left(\Omega_{+}\right)_{\text {loc }}^{2}$ solving Maxwell's equations in $\Omega_{+}$, satisfying the Silver-Müller radiation condition, and such that $\gamma_{\mathrm{T}}^{+} E=v$. The corresponding solution operator is continuous.

For interior problems we have

Theorem 2.2. There is a unique real positive strictly increasing and unbounded sequence $\left(k_{n}\right)$ such that with $\mathcal{K}=\left\{k_{n}: n \in \mathbb{N}\right\}$ we have

- for all $k \notin \mathcal{K}$, for all $v \in \mathrm{H}_{\mathrm{rot}}^{-1 / 2}(\Gamma)$ there is a unique $(E, H) \in \mathrm{H}_{\text {curl }}^{0}\left(\Omega_{-}\right)^{2}$ solving Maxwell's equations in $\Omega_{-}$and such that $\gamma_{\mathrm{T}}^{-} E=v$;

- for all $k \in \mathcal{K}$, the space of solutions $(E, H) \in \mathrm{H}_{\text {curl }}^{0}\left(\Omega_{-}\right)^{2}$ to Maxwell's equations in $\Omega_{-}$, such that $\gamma_{\mathrm{T}}^{-} E=0$, is a nonzero finite dimensional space.

The elements of $\mathcal{K}$ are called resonant wavenumbers. For any $k$, the vector space of electric fields $E$ in $\Omega_{-}$, such that with $H=1 /(i k Z) \operatorname{curl} E,(E, H)$ is in $\mathrm{H}_{\text {curl }}^{0}\left(\Omega_{-}\right)^{2}$ and solves Maxwell's equations in $\Omega_{-}$with the perfect conductor boundary condition $\gamma_{\mathrm{T}}^{-} E=0$, is denoted $\mathcal{E}_{k}$. It is finite dimensional and it is nonzero only if $k \in \mathcal{K}$.

Recall the Sommerfeld radiation condition

$$
\partial_{r} u-i k u=o(1 / r) \quad \text { with } r=|x|,
$$

and let $G_{k}$ be the fundamental solution of the Helmholtz operator $-\Delta-k^{2}$ satisfying it

$$
G_{k}(x, y)=\frac{e^{i k|x-y|}}{4 \pi|x-y|}
$$


Let $\Phi_{k}$ be the potential, mapping any sufficiently smooth tangent field $u$ on $\Gamma$ to the field in $\mathbb{R}^{3}$ defined away from $\Gamma$ by

$$
\left(\Phi_{k} u\right)(y)=\int_{\Gamma} G_{k}(x, y) u(x) \mathrm{d} x .
$$

Of fundamental importance to us will be the representation theorem ([29], Theorem 5.5.1, p. 234):

Theorem 2.3. Suppose $(E, H)$ is a field whose restrictions to $\Omega_{-}$and $\Omega_{+}$are in $\mathrm{H}_{\text {curl }}^{0}\left(\Omega_{-}\right)^{2}$ and $\mathrm{H}_{\text {curl }}^{0}\left(\Omega_{+}\right)_{\text {loc }}^{2}$ and solve Maxwell's equations for a given wavenumber $k$. Suppose also that it verifies the Silver-Müller radiation condition. Define the electric and magnetic currents $j$ and $m$ on $\Gamma$ by the jump formulas

$$
\begin{aligned}
j & =[H \times n]=\left(\gamma_{\mathrm{T}}^{-} H-\gamma_{\mathrm{T}}^{+} H\right) \times n, \\
m & =[E \times n]=\left(\gamma_{\mathrm{T}}^{-} E-\gamma_{\mathrm{T}}^{+} E\right) \times n .
\end{aligned}
$$

Then in $\Omega_{-}$and $\Omega_{+}$we have

$$
\begin{aligned}
E & =(+i k Z)\left(1+\left(1 / k^{2}\right) \operatorname{grad} \operatorname{div}\right) \Phi_{k} j+\operatorname{curl} \Phi_{k} m, \\
H & =(-i k / Z)\left(1+\left(1 / k^{2}\right) \operatorname{grad} \operatorname{div}\right) \Phi_{k} m+\operatorname{curl} \Phi_{k} j .
\end{aligned}
$$

Definition 2.4. For $k \neq 0$ we define the Electric Field Integral Operator, on tangent fields on $\Gamma$, by (the interior and exterior traces are equal)

$$
A_{k} u=\gamma_{T}\left(1+\left(1 / k^{2}\right) \operatorname{grad} \operatorname{div}\right) \Phi_{k} u .
$$

One shows that $A_{k}$ is continuous $\mathrm{H}_{\text {div }}^{s}(\Gamma) \rightarrow \mathrm{H}_{\text {rot }}^{s}(\Gamma)$. The EFIE for a given tangent field $v$ is the equation $A_{k} u=v$. From the preceding equations it follows that if $k \in \mathbb{R}_{+}^{*} \backslash \mathcal{K}, A_{k}: \mathrm{H}_{\text {div }}^{-1 / 2}(\Gamma) \rightarrow \mathrm{H}_{\mathrm{rot}}^{-1 / 2}(\Gamma)$ is invertible (29], Theorem 5.6.2, p. 247). But more interestingly we have the following results even if $k$ is a resonant wavenumber:

- for any $v \in \mathrm{H}_{\mathrm{rot}}^{-1 / 2}(\Gamma)$, if the interior problem with respect to $v$ has a solution $\left(E_{-}, H_{-}\right)$, then letting $\left(E_{+}, H_{+}\right)$be the exterior solution and putting $u=$ $\left(\gamma_{\mathrm{T}}^{-} H_{-}-\gamma_{\mathrm{T}}^{+} H_{+}\right) \times n$, we have $u \in \mathrm{H}_{\mathrm{div}}^{-1 / 2}(\Gamma)$ and $A_{k} u=v$;

- for any $v \in \mathrm{H}_{\mathrm{rot}}^{-1 / 2}(\Gamma)$, if we have a solution $u \in \mathrm{H}_{\mathrm{div}}^{-1 / 2}(\Gamma)$ to $A_{k} u=v$, then the solution $(E, H)$ of the exterior problem with respect to $v$ is given by

$$
E=\left(1-\left(1 / k^{2}\right) \operatorname{grad} \operatorname{div}\right) \Phi_{k} u, \quad H=1 /(i k Z) \operatorname{curl} \Phi_{k} u ;
$$

- for any $u \in \mathrm{H}_{\mathrm{div}}^{-1 / 2}(\Gamma), A_{k} u=0$ if and only if $u \in\left(\gamma_{\mathrm{T}}^{-} \operatorname{curl} \mathcal{E}_{k}\right) \times n$.

Thus for instance if $v$ is the tangential trace of a planar wave - which is the case in RCS computations - then the EFIE has a solution even at resonances, which is determined up to a finite dimensional space, and for any such solution the corresponding potential solves the exterior problem. One of the main goals of this paper is to determine to which extent an analogous property holds true for the discretized EFIE.

2.2. Variational formulation and discretization. Recall that the $\mathbb{C}$ bilinear form on tangent fields

$$
(u, v) \mapsto\langle u, v\rangle=\int_{\Gamma} u \cdot v
$$

induces a duality between $\mathrm{H}_{\mathrm{div}}^{-1 / 2}(\Gamma)$ and $\mathrm{H}_{\mathrm{rot}}^{-1 / 2}(\Gamma)$ ([29], Lemma 4.5.1, p. 208). 
For sufficiently smooth tangent fields we have (all integrals are on $\Gamma$ )

$$
\begin{aligned}
\left\langle A_{k} u, u^{\prime}\right\rangle= & \iint G_{k}(x, y) u(x) \cdot u^{\prime}(y) \mathrm{d} x \mathrm{~d} y \\
& -\left(1 / k^{2}\right) \iint G_{k}(x, y) \operatorname{div} u(x) \operatorname{div} u^{\prime}(y) \mathrm{d} x \mathrm{~d} y .
\end{aligned}
$$

In order to have a highest order term which is positive we will sometimes consider the opposite bilinear form (associated with $-A_{k}$ ). This leads to a variational formulation of the EFIE, also known as the Rumsey reaction principle. For a given $v \in \mathrm{H}_{\text {rot }}^{-1 / 2}(\Gamma)$ solve

$$
u \in \mathrm{H}_{\mathrm{div}}^{-1 / 2}(\Gamma) \quad \text { and } \quad \forall u^{\prime} \in \mathrm{H}_{\operatorname{div}}^{-1 / 2}(\Gamma) \quad\left\langle A_{k} u, u^{\prime}\right\rangle=\left\langle v, u^{\prime}\right\rangle .
$$

Whenever we have a family $\left(X_{h}\right)$ of subspaces of $\mathrm{H}_{\text {div }}^{-1 / 2}(\Gamma)$, the Galerkin method consists in considering the equations

$$
u \in X_{h} \quad \forall u^{\prime} \in X_{h} \quad\left\langle A_{k} u, u^{\prime}\right\rangle=\left\langle v, u^{\prime}\right\rangle
$$

and studying the convergence of the corresponding solutions (when they exist), with respect to the parameter $h$.

2.3. Some strategies for the analysis of the EFIE. As is clear from equation (2.22), the EFIE has dominant terms with different signs when restricted to gradients and divergence-free tangent fields. Therefore the theory of compact perturbations of coercive operators is not by itself enough to study the discretization of the EFIE.

It seems that the only numerical analysis available for the variational discretization of the EFIE is the original approach of Bendali [5], who introduces the charge density $q=\operatorname{div} u$ into the formulation, with Lagrange multipliers. More precisely the EFIE is formulated in the following way:

$$
\left\{\begin{array} { l } 
{ u \in \mathrm { H } _ { \mathrm { div } } ^ { - 1 / 2 } ( \Gamma ) } \\
{ q \in \mathrm { H } ^ { - 1 / 2 } ( \Gamma ) ^ { \bullet } }
\end{array} \quad \left\{\begin{array}{ll}
\forall u^{\prime} \in \mathrm{H}_{\mathrm{div}}^{-1 / 2}(\Gamma) & a\left(u, u^{\prime}\right)+b\left(q, u^{\prime}\right)=l\left(u^{\prime}\right), \\
\forall q^{\prime} \in \mathrm{H}^{-1 / 2}(\Gamma)^{\bullet} & c\left(q^{\prime}, u\right)+d\left(q, q^{\prime}\right)=0,
\end{array}\right.\right.
$$

where for any space $W$ of scalar functions on $\Gamma, W^{\bullet}$ denotes the subspace of $W$ whose elements are $\mathrm{L}^{2}$ orthogonal to the functions that are constant on each connected component of $\Gamma$, and

$$
\begin{aligned}
l\left(u^{\prime}\right) & =-\left\langle v, u^{\prime}\right\rangle, \\
a\left(u, u^{\prime}\right) & =-\iint G_{k}(x, y) u(x) \cdot u^{\prime}(y) \mathrm{d} x \mathrm{~d} y, \\
b\left(q, u^{\prime}\right) & =\left(1 / k^{2}\right) \iint G_{k}(x, y) q(x) \operatorname{div} u^{\prime}(y) \mathrm{d} x \mathrm{~d} y, \\
c\left(q^{\prime}, u\right) & =\left(1 / k^{2}\right) \iint G_{0}(x, y) q^{\prime}(x) \operatorname{div} u(y) \mathrm{d} x \mathrm{~d} y, \\
d\left(q, q^{\prime}\right) & =-\left(1 / k^{2}\right) \iint G_{0}(x, y) q(x) q^{\prime}(y) \mathrm{d} x \mathrm{~d} y .
\end{aligned}
$$

This formulation leads to an intricate mathematical analysis relying heavily upon the fact that the operator with kernel $G_{k}-G_{0}$ is of order -3 . Its main advantage is that for the usual Galerkin spaces $X_{h} \subset \mathrm{H}_{\text {div }}^{-1 / 2}(\Gamma)$ the spaces $W_{h}=\operatorname{div} X_{h}$ are simple subspaces of the usual Galerkin spaces of piecewise polynomial functions 
(with no continuity requirements). Thus the formulation (2.25) has a discrete counterpart on $W_{h} \times X_{h}$.

Nédélec [29] has used the following splitting of $X=\mathrm{H}_{\mathrm{div}}^{-1 / 2}(\Gamma)$ to prove the Fredholm property of the EFIE. Let $a$ be the bilinear form induced by the EFIE. Put $W=\mathrm{H}^{1 / 2}(\Gamma)$. Then $\operatorname{rot} W$ is a closed subspace of $X$. Let $V$ be the orthogonal of $\operatorname{rot} W$ with respect to $a$, i.e.,

$$
V=\{u \in X: \forall w \in W \quad a(\operatorname{rot} w, u)=0\} .
$$

Since the restriction of $a$ to $\operatorname{rot} W$ is a compact perturbation of a coercive form, $V \cap W$ is finite dimensional, and $V+W$ is closed and has finite codimension in $X$. Thus, up to finite dimensional spaces, one can search for the solution of the EFIE in the form $u=v+\operatorname{rot} w$, with $(v, w)$ solving

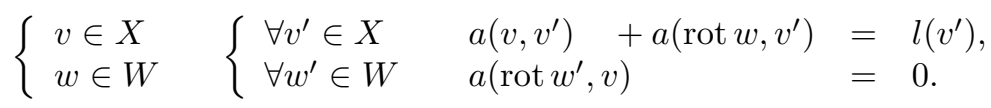

The conclusive remark is that on $V, a$ satisfies a Gårding inequality, thus this saddle-point problem is Fredholm of index 0.

2.4. Reformulation of the EFIE as a saddle-point. We will use a nonsymmetric variant of this last technique which has the advantage of not introducing the parasitic finite dimensional subspaces. In particular the analysis we propose does not need the fact that the operator with kernel $G_{k}-G_{0}$ is of order -3 , only that is it compact $\mathrm{H}^{-1 / 2}(\Gamma) \rightarrow \mathrm{H}^{1 / 2}(\Gamma)$.

In order to be more precise in our statements, we will require the theory of saddle-point problems. We assume familiarity with the theory of Inf-Sup conditions as presented for instance in Roberts-Thomas 34, and in particular the way discrete Inf-Sup conditions lead to convergence estimates for Galerkin methods. However, for completeness we include a result of Nicolaides [30, which generalizes the classical theorem of Brezzi [10].

Theorem 2.5. Let $X_{i}$ and $W_{i}$ for $i=1,2$ be Hilbert spaces. Let $a, b$ and $c$ be continuous bilinear forms on $X_{1} \times X_{2}, W_{1} \times X_{2}$ and $W_{2} \times X_{1}$. Let $V_{1}$ (resp. $V_{2}$ ) denote the right-hand kernel of $c$ on $W_{2} \times X_{1}$ (resp. $b$ on $W_{1} \times X_{2}$ ). Suppose that

$$
\begin{aligned}
& \inf _{V_{1}} \sup _{V_{2}} a \geq \alpha>0, \\
& \inf _{W_{1} \sup _{2} b \geq \beta}>0, \\
& \inf _{W_{2} \sup _{1} c \geq \gamma}>0, \\
& \forall v_{2} \in V_{2} \quad\left(\forall v_{1} \in V_{1} a\left(v_{1}, v_{2}\right)=0\right) \Rightarrow\left(v_{2}=0\right) .
\end{aligned}
$$

Then for all $(g, f) \in W_{2}^{\star} \times X_{2}^{\star}$ there is a unique $\left(w_{1}, u_{1}\right) \in W_{1} \times X_{1}$ such that

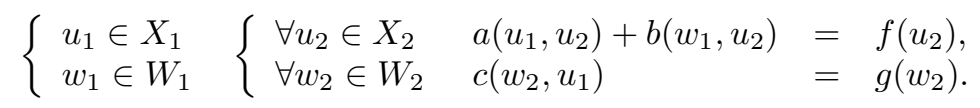

Moreover one has the continuity estimate for the solution operator

$$
\begin{aligned}
\left\|u_{1}\right\| & \leq \alpha^{-1}\|f\|+\gamma^{-1}\left(1+\alpha^{-1}\|a\|\right)\|g\|, \\
\left\|w_{1}\right\| & \leq \beta^{-1}\left(1+\alpha^{-1}\|a\|\right)\left(\|f\|+\gamma^{-1}\|a\|\|g\|\right) .
\end{aligned}
$$


If for all $(g, f)$ there is a unique solution $\left(w_{1}, u_{1}\right)$, then the above Inf-Sup conditions are satisfied for some $\alpha, \beta$ and $\gamma$, as noted by Bernardi et al. [6].

We return now to the special case of interest, the EFIE. We put $X=\mathrm{H}_{\text {div }}^{-1 / 2}(\Gamma)$ and let $a$ be the bilinear form on $X$ induced by the EFIE

$$
\begin{aligned}
a\left(u, u^{\prime}\right)= & \left(1 / k^{2}\right) \iint G_{k}(x, y) \operatorname{div} u(x) \operatorname{div} u^{\prime}(y) \mathrm{d} x \mathrm{~d} y \\
& -\iint G_{k}(x, y) u(x) \cdot u^{\prime}(y) \mathrm{d} x \mathrm{~d} y .
\end{aligned}
$$

Let $d$ be the bilinear form on $X$ defined by

$$
\begin{aligned}
d\left(u, u^{\prime}\right)= & -\left(1 / k^{2}\right) \iint G_{0}(x, y) \operatorname{div} u(x) \operatorname{div} u^{\prime}(y) \mathrm{d} x \mathrm{~d} y \\
& -\iint G_{0}(x, y) u(x) \cdot u^{\prime}(y) \mathrm{d} x \mathrm{~d} y .
\end{aligned}
$$

The involution of $X$ induced by the complex conjugation is denoted $u \mapsto \bar{u}$. Then it follows from the positivity of the operator with kernel $G_{0}$ that $-d(\cdot, \cdot)$ is a Hermitian scalar product on $X$. Thus for any closed subspace $X_{0}$ of $X$ and any closed subspace $W_{0}$ of $X_{0}$, if $X_{0}$ and $W_{0}$ are stable under conjugation (conjugation-stable for short) one has the orthogonal splitting $X_{0}=V_{0} \oplus W_{0}$, with

$$
V_{0}=\left\{u \in X_{0} \quad: \forall w \in W_{0} \quad d(w, u)=0\right\} .
$$

Put $W=\{u \in X: \operatorname{div} u=0\}$. Now let $X_{0}$ be any closed and conjugation-stable subspace of $X$, and put $W_{0}=X_{0} \cap W$, which is also closed and conjugation-stable. Then, for all $l \in X_{0}^{\star}, u$ solves

$$
u \in X_{0} \quad \text { and } \quad \forall u^{\prime} \in X_{0} \quad a\left(u, u^{\prime}\right)=l\left(u^{\prime}\right),
$$

if and only if $u=v+w$, with $(v, w)$ solving

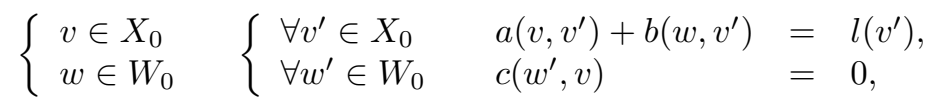

where $b$ is the restriction of $a$ to $W \times X$ and $c$ the restriction of $d$ to $W \times X$.

Consider first the case $X_{0}=X$. Let $\Theta: W \times X \rightarrow W^{\star} \times X^{\star}$ be the operator associated with the left-hand side of the above saddle-point (2.44). If the kernels $G_{k}$ are replaced by $G_{0}$ in $a$ and $b$ (keeping the outside coefficient $\left(1 / k^{2}\right)$ untouched), the corresponding operator $\Theta_{0}: W \times X \rightarrow W^{\star} \times X^{\star}$ is symmetric. It is the saddle-point mapping associated with the bilinear form $a_{0}$ defined by

$$
\begin{aligned}
a_{0}\left(u, u^{\prime}\right)= & \left(1 / k^{2}\right) \iint G_{0}(x, y) \operatorname{div} u(x) \operatorname{div} u^{\prime}(y) \mathrm{d} x \mathrm{~d} y \\
& -\iint G_{0}(x, y) u(x) \cdot u^{\prime}(y) \mathrm{d} x \mathrm{~d} y .
\end{aligned}
$$

For $\Theta_{0}$ the Brezzi compatibility estimates (2.34) and (2.35) are trivial. The cornerstone of the argument is

Theorem 2.6. On the right kernel of $c$ on $W \times X$, the bilinear form $a_{0}$ satisfies a Gårding inequality.

Proof. Let $V$ denote the right kernel of $c$ on $W \times X$. Remark first that $V$ is a supplementary of $\operatorname{ker}_{X} \operatorname{div}$ and that $\operatorname{div}: X \rightarrow \mathrm{H}^{-1 / 2}$ has closed range. It follows 
that div determines an isomorphism from $V$ to its range, so there is $C>0$ such that

$$
\forall v \in V \quad\|v\|_{X} \leq C|\operatorname{div} v|_{-1 / 2} .
$$

Hence the first term in the right-hand side of equation (2.45) is coercive on $V$.

Concerning the second term, notice that for elements $v$ of $V$ we have

$$
\operatorname{rot} \pi_{\mathrm{T}} \int G_{0}(x, y) v(x) \mathrm{d} x=0,
$$

where $\pi_{\mathrm{T}}$ denotes the orthogonal projection onto the tangent space. As shown in Nédélec [29], it follows that rot maps $V$ continuously into $\mathrm{H}^{-1 / 2}(\Gamma)$, hence $V$ is continuously imbedded in $\mathrm{H}_{\mathrm{T}}^{1 / 2}(\Gamma)$. In turn the injection $\mathrm{H}_{\mathrm{T}}^{1 / 2}(\Gamma) \rightarrow \mathrm{H}_{\mathrm{T}}^{-1 / 2}(\Gamma)$ is compact so the proof is complete.

From this it follows that $\Theta_{0}$ is Fredholm, and by symmetry its index must be 0 . Since $\Theta$ is a compact perturbation of $\Theta_{0}, \Theta$ is also Fredholm of index 0 .

If $u \mapsto a(u, \cdot)$ is injective, the above splitting, in the case $X_{0}=X$ shows that the map $\Theta$ is also injective. Therefore it is an isomorphism.

Remark 2.7. We could also have made the choice $W=\operatorname{rot} \mathrm{H}^{1 / 2}(\Gamma)$, which differs from the adopted one only by a finite dimensional space, whose elements are known as the Neumann fields (or harmonic fields). Some proofs would have to be modified.

\section{Inf-Sup ESTIMATES FOR THE EFIE}

We now turn to the discretization of the EFIE. Recall notation from Section 2.4. We consider the case where $X_{0}$ is a Galerkin space, one among a family $\left(X_{h}\right)$. Putting $W_{h}=X_{h} \cap W$, our strategy will be to first study the saddle-point problem associated with the EFIE on $W_{h} \times X_{h}$ and to give sufficient conditions on $\left(X_{h}\right)$ for it to satisfy uniform Inf-Sup estimates. From these we easily deduce Inf-Sup estimates for the original problem on $X_{h}$.

\subsection{Sufficient conditions for uniform Inf-Sup estimates.}

Lemma 3.1. Let $X$ be a Hilbert space. The scalar product is denoted (.|.), and orthogonality is denoted $\perp$. Let $X=V \oplus W$ be an orthogonal splitting. Let $\left(X_{h}\right)$ be an approximating family of closed subspaces. Put $W_{h}=W \cap X_{h}$, and $V_{h}=\{u \in$ $\left.X_{h}: u \perp W_{h}\right\}$. Then $\left(V_{h}\right)$ is approximating in $V$, and if $\left(V_{h}\right)$ is quasi-conforming in $V$, then $\left(W_{h}\right)$ is approximating in $W$.

Proof. Indeed the orthogonal projection $P_{h}$ onto $X_{h}$ maps $V$ into $V_{h}$, therefore $\left(V_{h}\right)$ is approximating. For any $w \in W$, put $P_{h} w=v_{h}+w_{h}$ with $v_{h} \in V_{h}$ and $w_{h} \in W_{h}$. We have

$$
\forall v^{\prime} \in V \quad\left(v_{h} \mid v_{h}\right)=\left(v_{h} \mid P_{h} w\right)=\left(v_{h} \mid w\right)=\left(v_{h}-v^{\prime} \mid w\right) .
$$

Hence

$$
\left\|v_{h}\right\|^{2} \leq\|w\|^{2} \sup _{v \in V_{h}} \inf _{v^{\prime} \in V}\left\|v-v^{\prime}\right\| /\|v\|,
$$

and since

$$
\left\|w-w_{h}\right\| \leq\left\|w-P_{h} w\right\|+\left\|v_{h}\right\|,
$$

it follows that if $\left(V_{h}\right)$ is quasi-conforming in $V$, then $\left\|w-w_{h}\right\|$ tends to 0 . 
Theorem 3.2. Recall notation from Section 2.4. Let $\left(X_{h}\right)$ be an approximating family of closed and conjugation-stable subspaces of $X$. Let $V$ be the right kernel of $c$ on $W \times X$ and $V_{h}$ the right kernel of $c$ on $W_{h} \times X_{h}$. If $\delta\left(V_{h}, V\right) \rightarrow 0$, then $\Theta$ is discrete LSF on $\left(W_{h} \times X_{h}\right) \times\left(W_{h} \times X_{h}\right)$, and $u \mapsto a(u, \cdot)$ is discrete LSF on $\left(X_{h} \times X_{h}\right)$.

Proof. We prove the result for $\Theta_{0}$. Then the theorem follows for $\Theta$ by Corollary 1.13, for $u \mapsto a_{0}(u, \cdot)$ by the equivalent splitting and for $u \mapsto a(u, \cdot)$ likewise.

We will use on $X$ the scalar product induced by $-d$. By Theorem 2.6, Example 1.14 and Lemma 1.19 it follows that if $N_{0}$ is the kernel of $a_{0}$ on $V \times V$, we have, for some $\alpha_{0}>0$ and $h_{0}>0$

$$
\forall h<h_{0} \inf _{N_{0}^{\perp} \cap V_{h}} \sup _{V_{h}} a_{0}>\alpha_{0},
$$

where $N_{0}^{\perp}$ is the orthogonal complement of $N_{0}$ in $X$.

For any $h$, let $V_{h}^{\perp}$ be the orthogonal complement of $V_{h}$ in $X_{h}$. Since $c$ is the restriction of $d$, we can choose a $\gamma>0$ such that

$$
\forall h \quad \gamma<\inf _{V_{h}^{\perp} W_{h}} \sup ^{t}=\inf _{W_{h}} \sup _{V_{h}^{\perp}} c=\inf _{W_{h}} \sup _{X_{h}} c .
$$

For any Banach space $Y$ with norm denoted $\|\cdot\|$, any continuous linear form $l$ on $Y$ and any subspace $Y_{h}$ of $Y$ we use the notation

$$
\|l\|_{Y_{h}}=\sup _{u \in Y_{h}}|l(u)| /\|u\| .
$$

Choose $h<h_{0}, w \in W_{h}, v \in N_{0}^{\perp} \cap V_{h}$ and $u \in V_{h}^{\perp}$. Put $(g, f)=\Theta_{0}(w, u+v)$. Following Nicolaides, one checks that

$$
\begin{aligned}
\|u\| & \leq \gamma^{-1}\left(\|g\|_{W_{h}}\right) \\
\|v\| & \leq \alpha_{0}^{-1}\left(\|f\|_{X_{h}}+\left\|a_{0}\right\|\|u\|\right), \\
\|w\| & \leq \gamma^{-1}\left(\|f\|_{X_{h}}+\left\|a_{0}\right\|\|v\|+\left\|a_{0}\right\|\|u\|\right) .
\end{aligned}
$$

Hence

$$
\begin{aligned}
\|u+v\| & \leq \alpha_{0}^{-1}\|f\|_{X_{h}}+\gamma^{-1}\left(1+\alpha_{0}^{-1}\left\|a_{0}\right\|\right)\|g\|_{W_{h}}, \\
\|w\| & \leq \gamma^{-1}\left(1+\alpha_{0}^{-1}\left\|a_{0}\right\|\right)\left(\|f\|_{X_{h}}+\gamma^{-1}\left\|a_{0}\right\|\|g\|_{W_{h}}\right) .
\end{aligned}
$$

For any subspace $M$ of $X$ let $P_{M}$ denote the orthogonal projection onto it. Note that

$$
\left(N_{0}^{\perp} \cap V_{h}\right)+V_{h}^{\perp}=\left\{u \in X_{h}: u \perp P_{V_{h}}\left(N_{0}\right)\right\},
$$

whereas

$$
N_{0}^{\perp} \cap X_{h}=\left\{u \in X_{h}: u \perp P_{X_{h}}\left(N_{0}\right)\right\} .
$$

Now since $N_{0} \subset V, \delta\left(V_{h}, V\right) \rightarrow 0$ and $V_{h}$ is approximating $V$, it follows that $N_{0}$, $P_{V_{h}}\left(N_{0}\right)$ and $P_{X_{h}}\left(N_{0}\right)$ are uniformly close to each other (all the gaps tend to 0 ). Hence $\left(N_{0}^{\perp} \cap V_{h}\right)+V_{h}^{\perp}$ is uniformly close to $N_{0}^{\perp} \cap X_{h}$ and therefore we deduce from the above that

$$
\liminf _{h} \inf _{W_{h} \times\left(N_{0}^{\perp} \cap X_{h}\right)} \sup _{W_{h} \times X_{h}} \Theta_{0}>0 .
$$

Considering that $W$ and $X$ are orthogonal subspaces of the product space, the theorem follows. 
We will give some explicit examples of such Galerkin spaces in Section 3.5.

3.2. Spurious electric currents. Suppose for the moment that the hypotheses of Theorem 3.2 is verified, so that for any nonzero wavenumber $k$ the associated bilinear form $a_{k}$ is discrete LSF on $\left(X_{h} \times X_{h}\right)$. Let $I$ be a compact interval of positive reals (which might very well contain resonant wavenumbers). Since the map $k \mapsto a_{k}$ is continuous, it maps $I$ to a compact set, and we can apply Corollary 1.17, which we express in terms of sequences:

Suppose we have for each integer $n$, a wavenumber $k_{n} \in I$, a discretization parameter $h_{n}$ and a current $u_{n} \in X_{h_{h}}$, such that $\left(h_{n}\right)$ tends to 0 and

$$
\sup _{u^{\prime} \in X_{h_{n}}} \frac{\left|a_{k_{n}}\left(u_{n}, u^{\prime}\right)\right|}{\left\|u^{\prime}\right\|_{-1 / 2}}=o\left(\left\|u_{n}\right\|_{-1 / 2}\right) .
$$

Then we have (the constant $C$ stems from the standard duality $\langle\cdot, \cdot\rangle$ between $\mathrm{H}_{\text {rot }}^{-1 / 2}(\Gamma)$ and $\mathrm{H}_{\text {div }}^{-1 / 2}(\Gamma)$ and is of course independent of the wavenumber)

$$
\left\|A_{k_{n}} u_{n}\right\|_{\mathrm{H}_{\mathrm{rot}}^{-1 / 2}(\Gamma)} \leq C \sup _{u^{\prime} \in X} \frac{\left|a_{k_{n}}\left(u_{n}, u^{\prime}\right)\right|}{\left\|u^{\prime}\right\|_{-1 / 2}}=o\left(\left\|u_{n}\right\|_{-1 / 2}\right) .
$$

From the uniform continuity of the solution operator for the exterior problem on $I$, we deduce that the electromagnetic field generated by $u_{n}$ is negligible compared with $\left\|u_{n}\right\|_{-1 / 2}$. More precisely if $\left(E_{n}, H_{n}\right)$ is given by

$$
E_{n}=\left(1-\left(1 / k_{n}^{2}\right) \operatorname{grad} \operatorname{div}\right) \Phi_{k_{n}} u_{n}, \quad H_{n}=1 /\left(i k_{n} Z\right) \operatorname{curl} \Phi_{k_{n}} u_{n},
$$

then for any $R$,

$$
\left\|E_{n}\right\|_{\mathrm{H}_{\mathrm{curl}}^{0}\left(\Omega_{+} \cap B_{R}\right)}=o\left(\left\|u_{n}\right\|_{-1 / 2}\right),
$$

and a similar estimate holds for $H_{n}$. Expressed differently, if we have bounded currents producing small discrete residual errors, then the near-fields they radiate are also small. The far-field pattern associated with an exterior electromagnetic field depends continuously upon the boundary data, so it is also negligible compared with $\left\|u_{n}\right\|_{-1 / 2}$.

If one looks at a single wavenumber of interest (for instance a resonant one), then by Remark [1.16] one can exhibit rather explicit estimates as follows: Suppose $k$ is a resonant frequency, put $a=a_{k}$, and let $l$ be a linear form which is a compatible right-hand side. Let $u$ denote a solution

$$
\forall v \in X \quad a(u, v)=l(v) .
$$

Let $u_{h}^{b}$ be the best approximation of $u$ in $X_{h}$, and suppose we solve the Galerkin equation with an error $\epsilon_{h}$

$$
\sup _{v \in Y_{h}}\left|a\left(u_{h}, v\right)-l(v)\right| /\|v\| \leq \epsilon_{h} .
$$

We also have

$$
\sup _{v \in Y_{h}}\left|a\left(u_{h}^{b}, v\right)-l(v)\right| /\|v\| \leq\|a\|\left\|u_{h}^{b}-u\right\| .
$$

Hence

$$
\sup _{v \in Y_{h}}\left|a\left(u_{h}-u_{h}^{b}, v\right)\right| /\|v\| \leq \epsilon_{h}+\|a\|\left\|u_{h}^{b}-u\right\| .
$$


Let $N$ denote the (right) kernel of $a$. Let $\delta_{h}$ be the order of best approximation of the elements of $N$ by elements of $X_{h}$. Then by equation (1.49) we have the estimate

$$
\inf _{v \in N}\left\|u_{h}-u_{h}^{b}-v\right\| \leq C\left(\epsilon_{h}+\left\|u_{h}^{b}-u\right\|+\delta_{h}\left\|u_{h}-u_{h}^{b}\right\|\right) .
$$

As will be seen later, for the standard Finite Element approximation, $\delta_{h}$ and $\left\|u_{h}^{b}-u\right\|$ are of order $h^{3 / 2}$, and an $\epsilon_{h}$ of this order is also possible. In other words the discrete Galerkin solution can be written as a best approximation, plus an element of the kernel (which does not radiate at all), plus a current of magnitude $h^{3 / 2}$ less than $\left\|u_{h}\right\|$.

Notice however that for an $\epsilon_{h}$ of order $h^{3 / 2},\left\|u_{h}\right\|$ can be bounded, but if one asks for too small an $\epsilon_{h}$ (smaller than $h^{3 / 2}$ ), then $\left\|u_{h}\right\|$ might be forced to be very big.

3.3. Sufficient conditions in integer exponent Sobolev spaces. The preceding sufficient conditions for the good behavior of the EFIE were formulated in half-integer Sobolev norms. We prove here that these condition hold under some hypotheses formulated in the more familiar integer Sobolev norms.

Recall notation from Section 2.4. Consider the following hypotheses for a family of spaces $X_{h}$ :

(H0) The spaces $X_{h}$ are finite dimensional conjugation-stable subspaces of $\mathrm{H}_{\text {div }}^{0}(\Gamma)$.

(H1) There is $C>0$ such that for all $u \in \mathrm{H}_{\text {div }}^{1}(\Gamma)$

$$
\inf _{u^{\prime} \in X_{h}}\left\|u-u^{\prime}\right\|_{0} \leq C h\|u\|_{1} \text {. }
$$

(H2) There is $C>0$ such that for all $u \in X_{h},\|u\|_{0} \leq C h^{-1}\|u\|_{-1}$.

(H3) There is $C>0$ such that for all $u \in X_{h}$ if

$$
\forall w \in W_{h} \quad\langle u, w\rangle=0,
$$

then the solution $p$ of

$$
p \in \mathrm{H}^{1}(\Gamma)^{\bullet} \quad \text { and } \quad \Delta p=\operatorname{div} u
$$

satisfies

$$
|u-\operatorname{grad} p|_{0} \leq C h|\operatorname{div} u|_{0} .
$$

Notice that by (H3) we have the usual Inf-Sup estimate: There is $C>0$ such that

$$
\inf _{q \in \operatorname{div} X_{h}} \sup _{u \in X_{h}} \frac{|\langle q, \operatorname{div} u\rangle|}{|q|_{0}\|u\|_{0}} \geq \frac{1}{C} .
$$

Let $\Omega_{h}: \mathrm{H}_{\mathrm{div}}^{0} \rightarrow X_{h}$ map $u_{0} \in \mathrm{H}_{\mathrm{div}}^{0}$ to $u \in X_{h}$, the solution of

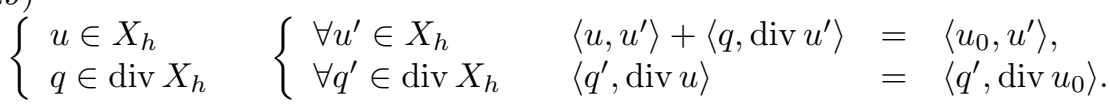

Under the above hypotheses one has an estimate of the form, for $u_{0} \in \mathrm{H}_{\text {div }}^{1}$,

$$
\left\|\Omega_{h} u_{0}-u_{0}\right\|_{0} \leq C h\left\|u_{0}\right\|_{1} .
$$

Moreover $\Omega_{h}$ maps divergence-free fields to divergence-free fields.

We denote by $V$ the right kernel of $c$ on $W \times X$ and by $V_{h}$ the one on $W_{h} \times X_{h}$. 
Theorem 3.3. Under the above hypotheses $\left(V_{h}\right)$ is quasi-conforming in $V$. More precisely there is $C$ such that for all $h$ and all $v \in V_{h}$, if $p$ solves

$$
p \in \mathrm{H}^{1}(\Gamma)^{\bullet} \text { and } \Delta p=\operatorname{div} v
$$

and $w$ solves

$$
w \in W \quad \forall w^{\prime} \in W \quad d\left(w, w^{\prime}\right)=-d\left(\operatorname{grad} p, w^{\prime}\right),
$$

then $\operatorname{grad} p+w \in V$ and

$$
\|v-(\operatorname{grad} p+w)\|_{-1 / 2} \leq C h^{1 / 2}\|v\|_{-1 / 2} .
$$

Proof. Put $v=v_{0}+v_{1}$ with $v_{0} \in W_{h}$ and $v_{1} \in X_{h}$ such that $\forall w \in W_{h}\left\langle w, v_{1}\right\rangle=0$. According to (H3) we have

$$
\left\|v_{1}-\operatorname{grad} p\right\|_{0} \leq C h\|u\|_{0} .
$$

So from (H2) we deduce

$$
\left\|v_{1}-\operatorname{grad} p\right\|_{-1 / 2} \leq C h^{1 / 2}\|u\|_{-1 / 2} .
$$

We will use a regularity result that we state without proof. Let $\Lambda$ be the operator on tangent fields that maps any $u$ to a divergence-free $w$ such that for all divergencefree $w^{\prime}$ we have $d\left(w, w^{\prime}\right)=d\left(u, w^{\prime}\right)$. Then $\Lambda$ is continuous $\mathrm{H}_{\mathrm{T}}^{s}(\Gamma) \rightarrow \mathrm{H}_{\mathrm{T}}^{s}(\Gamma)[$

The field $w$ is defined by $w=-\Lambda \operatorname{grad} p$. From the $\mathrm{H}_{\mathrm{T}}^{1}$ continuity of $\Lambda$, it follows that

$$
\left\|\Omega_{h} w-w\right\|_{0} \leq C h\|w\|_{1}=C h|w|_{1} \leq C h|\operatorname{grad} p|_{1} \leq C h\|u\|_{0} .
$$

Hence

$$
\left\|\Omega_{h} w-w\right\|_{-1 / 2} \leq C h^{1 / 2}\|u\|_{-1 / 2} .
$$

But for all $w^{\prime} \in W_{h}$

$$
d\left(\Omega_{h} w-v_{0}, w^{\prime}\right)=d\left(\Omega_{h} w+v_{1}, w^{\prime}\right)=d\left(\left(\Omega_{h} w-w\right)+\left(v_{1}-\operatorname{grad} p\right), w^{\prime}\right) .
$$

The absolute value of this last expression is bounded by

$$
C\left(\left\|\Omega_{h} w-w\right\|_{-1 / 2}+\left\|v_{1}-\operatorname{grad} \phi\right\|_{-1 / 2}\right)\left\|w^{\prime}\right\|_{-1 / 2} .
$$

Taking $w^{\prime}$ to be the conjugate of $\Omega_{h} w-v_{0} \in W_{h}$ gives

$$
\left\|\Omega_{h} w-v_{0}\right\|_{-1 / 2} \leq C h^{1 / 2}\|u\|_{-1 / 2} .
$$

Combining equation (3.35) with (3.37) and (3.40) gives the theorem.

\footnotetext{
${ }^{1}$ This regularity result is comparable to the following more standard one. The pseudodifferential operator $A$ defined by

$$
\left\langle A \varphi, \varphi^{\prime}\right\rangle=\iint \frac{1}{|x-y|} \operatorname{rot} \varphi(x) \cdot \operatorname{rot} \varphi^{\prime}(y) \mathrm{d} x \mathrm{~d} y
$$

is an isomorphism of order 1 , and the pseudo-differential operator $B$ defined by

$$
\left\langle B \varphi, \varphi^{\prime}\right\rangle=\iint \frac{1}{|x-y|} \operatorname{grad} \varphi(x) \cdot \operatorname{rot} \varphi^{\prime}(y) \mathrm{d} x \mathrm{~d} y
$$

is a morphism of order less that 1 (one can show that it is of order 0 ). The operator $A^{-1} B$ is therefore of order less than 0 .
} 
3.4. Convergence rates. Let $\left(X_{h}\right)$ be a family of Galerkin spaces satisfying (H0) and (H1). Let $\mathcal{Q}_{h}$ be the $\mathrm{H}_{\text {div }}^{0}(\Gamma)$-orthogonal projection onto $X_{h}$. Then we have

$$
\left\|\mathcal{Q}_{h} u\right\|_{0} \leq C\|u\|_{0} \text { and }\left\|u-\mathcal{Q}_{h} u\right\|_{0} \leq C h\|u\|_{1} .
$$

The spaces $\mathrm{H}_{\text {div }}^{s}(\Gamma)$ for $0 \leq s \leq 1$ can be obtained by interpolation. Hence interpolation on the operator $\mathcal{I}-\mathcal{Q}_{h}$, for $0 \leq s \leq 1$, gives

$$
\left\|u-\mathcal{Q}_{h} u\right\|_{0} \leq C h^{s}\|u\|_{s}
$$

Then one uses the regularity of the $\mathrm{H}_{\text {div }}^{0}(\Gamma)$-inner product (written $\left.(\cdot \mid \cdot)_{0}\right)$ on various Sobolev spaces. This technique is the familiar Aubin-Nitsche trick. That $\mathrm{H}_{\text {div }}^{s}(\Gamma)$ and $\mathrm{H}_{\text {div }}^{-s}(\Gamma)$ are dual with respect to the $\mathrm{H}_{\text {div }}^{0}(\Gamma)$-inner product can be deduced from the fact that the operator $I$ - grad div is an isomorphism $\mathrm{H}_{\text {div }}^{s}(\Gamma) \rightarrow \mathrm{H}_{\text {rot }}^{s-1}(\Gamma)$ and that this space, as already mentioned, is the $\mathrm{L}_{\mathrm{T}}^{2}$-dual of $\mathrm{H}_{\text {div }}^{-s}(\Gamma)$. Both of these facts can be proved using the Helmholtz decomposition and regularity of the Laplacian. For $0 \leq s \leq 1$ we have

$$
\begin{aligned}
\left\|u-\mathcal{Q}_{h} u\right\|_{-s} & \leq C \sup _{v \in \mathrm{H}_{\mathrm{div}}^{s}} \frac{\left|\left(u-\mathcal{Q}_{h} u \mid v\right)_{0}\right|}{\|v\|_{s}} \\
& \leq C \sup _{v \in \mathrm{H}_{\mathrm{div}}^{s}} \frac{\left|\left(u-\mathcal{Q}_{h} u \mid v-\mathcal{Q}_{h} v\right)_{0}\right|}{\|v\|_{s}} \\
& \leq C\left\|u-\mathcal{Q}_{h} u\right\|_{0}\left\|\mathcal{I}-\mathcal{Q}_{h}\right\|_{0, s} .
\end{aligned}
$$

Here $\left\|\mathcal{I}-\mathcal{Q}_{h}\right\|_{0, s}$ is of course the norm of the induced map

$$
\mathcal{I}-\mathcal{Q}_{h}: \mathrm{H}_{\mathrm{div}}^{s}(\Gamma) \rightarrow \mathrm{H}_{\mathrm{div}}^{0}(\Gamma) .
$$

This gives for $0 \leq s, s^{\prime} \leq 1$

$$
\left\|u-\mathcal{Q}_{h} u\right\|_{-s} \leq C h^{s+s^{\prime}}\|u\|_{s^{\prime}} .
$$

For smooth scatterers and smooth incident waves (such as plane waves) the solution $u$ of the EFIE is known to be smooth, and in particular $u \in \mathrm{H}_{\text {div }}^{1}(\Gamma)$. Since the Inf-Sup condition yields quasi-optimal convergence, we therefore have

Theorem 3.4. Under the above hypotheses the Galerkin solution $u_{h}$ of the EFIE satisfies the convergence estimate $\left\|u-u_{h}\right\|_{-1 / 2} \leq C h^{3 / 2}$.

3.5. Some well-known spaces. Let $\wp$ be the orthogonal projection onto $\Gamma$, which is defined and smooth on a tubular neighborhood of $\Gamma$. Let $\left(\mathcal{T}_{h}\right)$ be a family of triangulations of $\Gamma$, where for all $h$ the largest diameter of a triangle of $\mathcal{T}_{h}$ is $h$. We will always suppose that $\left(\mathcal{T}_{h}\right)$ has the minimum angle property. Let $\Gamma_{h}$ be the affine polyhedron determined by $\mathcal{T}_{h}$, considered as a Lipschitz manifold. For small enough $h, \wp$ induces Lipschitz-isomorphisms $\Gamma_{h} \rightarrow \Gamma$, and we denote by $\Xi_{h}$ the inverse mappings.

Fix a nonzero $m \in \mathbb{N}$. On $\Gamma_{h}$ we consider the space $S^{0}\left(\mathcal{T}_{h}\right)$ of continuous scalar functions whose restriction to any triangle is $P^{m}$ (a polynomial of degree $m$ ), the space $\mathrm{S}^{1}\left(\mathcal{T}_{h}\right)$ of Raviart-Thomas $\mathrm{H}_{\text {div }}^{0}$ conforming vector fields of degree $m$, and the space $\mathrm{S}^{2}\left(\mathcal{T}_{h}\right)$ of scalar functions whose restriction to any triangle is $P^{m-1}$.

From these finite element spaces on $\Gamma_{h}$ we deduce finite element spaces on $\Gamma$ by the transport formulas

$$
\begin{array}{rrr}
\mathrm{S}_{h}^{0} & =\{x \mapsto & \left.p\left(\Xi_{h}(x)\right): p \in \mathrm{S}^{0}\left(\mathcal{T}_{h}\right)\right\}, \\
\mathrm{S}_{h}^{1}= & \{x \mapsto & \left.\operatorname{Jac} \Xi_{h}(h) \Xi_{h}(x)^{-1} u\left(\Xi_{h}(x)\right): u \in \mathrm{S}^{1}\left(\mathcal{T}_{h}\right)\right\}, \\
\mathrm{S}_{h}^{2} & =\{x \mapsto & \left.\operatorname{Jac} \Xi_{h}(x) q\left(\Xi_{h}(x)\right): q \in \mathrm{S}^{2}\left(\mathcal{T}_{h}\right)\right\} .
\end{array}
$$


These transport formulas were chosen to make the following diagram commute. The horizontal arrows are the differential operators rot and div, whereas the vertical ones are the above transport formulas.

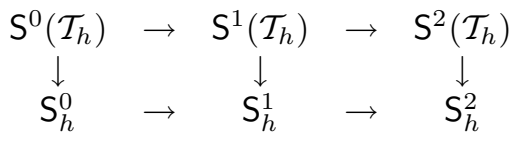

If we wish to be precise about the order $m$, we use superscripts $S_{h}^{0, m}, S_{h}^{1, m}$ and $\mathrm{S}_{h}^{2, m-1}$.

It is well known that $\left(\mathrm{S}_{h}^{1}\right)$ satisfies (H1). If the mesh is quasi-uniform, then (H2) also holds. The only remaining point is (H3). This property has been found to be very important in the study of eigenvalue problems by mixed formulations; see in particular Boffi et al. [8] and Demkowicz et al. 20]. This is because it implies a discrete compactness result which in the three-dimensional setting is due to Kikuchi [24]. Since this property is equally important to the present problem, we include a short proof of it. It goes without saying that the absence of a boundary on $\Gamma$ greatly simplifies our task. Ramifications can be found in Boffi 7 .

The usual degrees of freedom pertaining to $\mathcal{T}_{h}$ on $\Gamma_{h}$ can be transported to the curved triangles $\Xi_{h}^{-1}(T)$ for $T \in \mathcal{T}_{h}$. With these, an interpolation operator $\Pi_{h}$ onto $\mathrm{S}_{h}^{1}$ can be defined on spaces with slightly stronger regularity than $\mathrm{H}_{\text {div }}^{0}(\Gamma)$; see Brezzi-Fortin [12, p. 125, and Roberts-Thomas [34, p. 549, for two different variants in the planar setting. We will use the following properties of this interpolation operator:

- $\Pi_{h}$ is a projector onto $\mathrm{S}_{h}^{1}$ defined on some extension of $\mathrm{H}_{\mathrm{T}}^{1}(\Gamma)+\mathrm{S}_{h}^{1}$;

- $\Pi_{h}$ maps divergence-free fields to divergence-free fields;

- $\exists C>0 \forall u \in \mathrm{H}_{\mathrm{T}}^{1}(\Gamma) \forall h \quad\left|u-\Pi_{h} u\right|_{0} \leq C h|u|_{1}$.

Theorem 3.5. For any regular family of triangulations $\left(\mathcal{T}_{h}\right),\left(\mathrm{S}_{h}^{1}\right)$ satisfies $(\mathrm{H} 3)$, i.e., with $X_{h}=\mathrm{S}_{h}^{1}$, there is $C>0$ such that for all $u \in X_{h}$ if

$$
\forall w \in W_{h} \quad\langle u, w\rangle=0,
$$

then the solution $p$ of

$$
p \in \mathrm{H}^{1}(\Gamma)^{\bullet} \quad \Delta p=\operatorname{div} u
$$

satisfies

$$
|u-\operatorname{grad} p|_{0} \leq C h|\operatorname{div} u|_{0} .
$$

Proof. Note that $\operatorname{div}(\operatorname{grad} p-u)=0$, so

$$
\operatorname{div}\left(\Pi_{h} \operatorname{grad} p-u\right)=\operatorname{div} \Pi_{h}(\operatorname{grad} p-u)=0 .
$$

Put $\tilde{u}=\Pi_{h} \operatorname{grad} p$. The approximation property of $\Pi_{h}$ and the regularity of the Laplacian yield

$$
|\tilde{u}-\operatorname{grad} p|_{0} \leq C h|\operatorname{grad} p|_{1} \leq C h|\operatorname{div} u|_{0} .
$$

Moreover for any divergence-free $u^{\prime} \in X_{h}$ we have

$$
\left\langle\tilde{u}-u, u^{\prime}\right\rangle=\left\langle\tilde{u}, u^{\prime}\right\rangle=\left\langle\tilde{u}-\operatorname{grad} p, u^{\prime}\right\rangle .
$$

Applying this identity with $u^{\prime}$, the conjugate of $\tilde{u}-u$ gives

$$
|\tilde{u}-u|_{0} \leq|\tilde{u}-\operatorname{grad} p|_{0} \leq C h|\operatorname{div} u|_{0} .
$$


Therefore we have

$$
|u-\operatorname{grad} p|_{0} \leq|u-\tilde{u}|_{0}+|\tilde{u}-\operatorname{grad} p|_{0} \leq C h|\operatorname{div} u|_{0} .
$$

\section{ACKNOWLEDGMENTS}

I am indebted to F. Béreux for the important empirical observation that at resonant frequencies, the spurious currents do not pollute the computed far-field pattern. Also this work would not have been possible had I not received the invaluable advice of J.-C. Nédélec.

\section{REFERENCES}

[1] T. Abboud, Etude mathématique et numérique de quelques problèmes de diffraction d'ondes électromagnétiques, $\mathrm{PhD}$ thesis, Ecole Polytechnique, 1991.

[2] I. Babuska, Error bounds for the finite element method, Numer. Math., Vol. 16, pp. 322-333, 1971. MR 44:6166

[3] I. Babuska, A.K. Aziz, Survey lectures on the mathematical foundations of the finite element method, pp. 3-359, in A.K. Aziz (ed.), "The mathematical foundations of the finite element method with applications to partial differential equations", Academic Press, New York, 1972. MR 54:9111

[4] I. Babuska, J. Osborn, Eigenvalue problems, pp. 641-788, in P.G. Ciarlet, J.-L. Lions (eds.), "Handbook of numerical analysis, Vol. II, Finite element methods (Part 1)", North-Holland, 1991.

[5] A. Bendali, Numerical analysis of the exterior boundary value problem for the time-harmonic Maxwell equations by a boundary finite element method, Part 1: The continuous problem, Math. Comp., Vol. 43, No. 167, pp. 29-46, 1984, Part 2: The discrete problem, Math. Comp., Vol. 43, No. 167, pp. 47-68, 1984. MR 86i:65071a MR 86i:65071b

[6] C. Bernardi, C. Canuto, Y. Maday, Generalized Inf-Sup conditions for the Chebyshev spectral approximation of the Stokes problem, SIAM J. Numer. Anal., Vol. 25, No. 6, pp. 1237-1271, 1988. MR 90e:65151

[7] D. Boffi, Discrete compactness and Fortin operator for edge elements, Numer. Math., Vol. 87, No. 2, pp. 229-246, 2000. MR 2001k:65168

[8] D. Boffi, F. Brezzi, L. Gastaldi, On the problem of spurious eigenvalues in the approximation of linear elliptic problems in mixed form, Math. Comp., Vol. 69, No. 229, pp. 121-140, 1999. MR 2000i: 65175

[9] A. DeLaBourdonnaye, Décomposition de $\mathrm{H}_{\mathrm{div}}^{-1 / 2}(\Gamma)$ et nature de l'opérateur de SteklovPoincaré du problème extérieur de l'électromagnétisme, C. R. Acad. Sci. Paris Sér. I Math., Vol. 316, No. 4, pp. 369-372, 1993. MR 93k:78004

[10] F. Brezzi, On the existence, uniqueness and approximation of saddle-point problems arising from Lagrangian multipliers, RAIRO Anal. Numér., Vol. 8, No. R-2, pp. 129-151, 1974. MR 51:1540

[11] F. Brezzi, J. Douglas (Jr.), L.D. Marini, Two families of mixed finite elements for second order elliptic problems, Numer. Math., Vol. 47, pp. 217-235, 1985. MR 87g:65133

[12] F. Brezzi, M. Fortin, Mixed and hybrid finite element methods, Springer-Verlag, 1991. MR 92d:65187

[13] A. Buffa, M. Costabel, C. Schwab, Boundary element methods for Maxwell's equations on non-smooth domains, Numer. Math., Vol. 92, No. 4, pp. 679-710, 2002.

[14] M. Cessenat, Mathematical methods in electromagnetism, Linear theory and applications, World Scientific Publishing Co., River Edge, NJ, 1996. MR 97j:78001

[15] S.H. Christiansen, F. Béreux, J.-C. Nédélec, J.-P. Martinaud, Algorithme de simulation électromagnétique, notamment des performances d'une antenne, Patent by Thomson-CSF Detexis, Reg. No. 0007456 at INPI, dated June 9, 2000.

[16] S.H. Christiansen, J.-C. Nédélec, Des préconditionneurs pour la résolution numérique des équations intégrales de frontière de l'électromagnétisme, C. R. Acad. Sci. Paris, Sér. I Math., Vol. 331, No. 9, pp. 733-738, 2000. MR 2001i:78022

[17] D.L. Colton, R. Kress, Integral equation methods in scattering theory, John Wiley \& Sons, New York, 1983. MR 85d:35001 
[18] M. Costabel, Boundary integral operators on Lipschitz domains: elementary results, SIAM J. Math. Anal., Vol. 19, No. 3, pp. 613-626, 1988. MR 89h:35090

[19] L. Demkowicz, Asymptotic convergence in finite and boundary element methods, Part 1, Theoretical results, Comput. Math. Appl., Vol. 27, No. 12, pp. 69-84, 1994, Part 2, The LBB constant for rigid and elastic scattering problems, Comput. Math. Appl., Vol. 28, No. 6, pp. 93-109, 1994. MR 95h:65080, MR 95k:65106

[20] L. Demkowicz, P. Monk, C. Schwab, L. Vardapetyan, Maxwell eigenvalues and discrete compactness in two dimensions, Comput. Math. Appl., Vol. 40, No. 4-5, pp. 589-605, 2000. MR 2002e:65166

[21] G.C. Hsiao, R.E. Kleinman, Mathematical foundations of error estimates in numerical solutions of integral equations in electromagnetics, IEEE Trans. Ant. Prop., Vol. 47, No. 3, pp. 316-328, 1997. MR 98a:78001

[22] J.D. Jackson, Classical electrodynamics, Second edition, John Wiley \& Sons, New YorkLondon-Sydney, 1975. MR 55:9721

[23] T. Kato, Perturbation theory for linear operators, Second edition, Springer-Verlag, BerlinNew York 1976. MR 53:11389

[24] F. Kikuchi, On a discrete compactness property for the Nédélec finite elements, J. Fac. Sci. Univ. Tokyo, Sect. 1A Math., Vol. 36, pp. 479-490, 1989. MR 91h:65173

[25] J.-L. Lions, E. Magenes, Problèmes aux limites non homogènes et applications, Vol. I, Vol. II, Dunod, Paris, 1968. MR 40:512, MR 40:513

[26] J.-C. Nédélec, Curved finite element methods for the solution of singular integral equations on surfaces in $\mathbb{R}^{3}$, Comput. Methods Appl. Mech. Engrg., Vol. 8, No. 1, pp. 61-80, 1976. MR 56:13741

[27] J.-C. Nédélec, Computation of eddy currents on a surface in $\mathbb{R}^{3}$ by finite element methods, SIAM J. Numer. Anal., Vol. 15, No. 3, pp. 580-594, 1978. MR 58:14409

[28] J.-C. Nédélec, Eléments finis mixtes incompressibles pour l'équation de Stokes dans $\mathbb{R}^{3}$, Numer. Math., Vol. 39, pp. 97-112, 1982. MR 83g:65111

[29] J.-C. Nédélec, Acoustic and Electromagnetic Equations, Integral Representations for Harmonic Problems, Springer-Verlag, 2001. MR 2002c:35003

[30] R.A. Nicolaides, Existence, uniqueness and approximation for generalized saddle point problems, SIAM J. Numer. Anal., Vol. 19, No. 2, pp. 349-357, 1982. MR 83d:49023

[31] L. Paquet, Problèmes mixtes pour le système de Maxwell, Ann. Fac. Sci. Toulouse Math., Vol. 4, No. 2, pp. 103-141, 1982. MR 84e:58075

[32] S.S.M. Rao, D.R. Wilton, A.W. Glisson, Electromagnetic scattering by surfaces of arbitrary shape, IEEE Trans. Ant. Prop. AP-30, pp. 409-418, 1982.

[33] P.A. Raviart, J.-M. Thomas, A mixed finite element method for 2 nd order elliptic problems, pp. 292-315, in I. Galligani, E. Magenes (eds.), "Mathematical aspects of the finite element method", Lecture Notes in Math., Vol. 606, Springer-Verlag, Berlin and New York, 1977. MR 58:3547

[34] J.E. Roberts, J.-M. Thomas, Mixed and hybrid methods, pp. 523-640, in P.G. Ciarlet, J.-L. Lions (eds.), "Handbook of numerical analysis, Vol. II, Finite element methods (Part 1)", North-Holland, 1991.

[35] W. Rudin, Functional analysis, Second edition, McGraw-Hill, New York, 1991. MR 92k:46001

[36] A.H. Schatz, An observation concerning Ritz-Galerkin methods with indefinite bilinear forms, Math. Comp., Vol. 28, pp. 959-962, 1974. MR 51:9526

[37] G. Strang, Variational crimes in the finite element method, pp. 689-710, in A.K. Aziz (ed.), "The mathematical foundations of the finite element method with applications to partial differential equations", Academic Press, New York, 1972. MR 54:1668

[38] M. Taylor, Partial differential equations, Vol. I Basic theory, Vol. II Qualitative studies in linear equations, Springer-Verlag, New York, 1996. MR 98b:35002b, MR 98b:35003

[39] I. Terrasse, Résolution mathématique et numérique des équations de Maxwell instationnaires par une méthode de potentiels retardés, PhD thesis, Ecole Polytechnique, 1993.

[40] W.L. Wendland, Strongly elliptic boundary integral equations, pp. 511-561, in A. Iserles, M. Powell (eds.), "The state of the art in numerical analysis (Birmingham 1986)", Inst. Math. Appl. Conf. Ser. New Ser. 9, Oxford Univ. Press, New York, 1987. MR 88m:65209

Matematisk Institutt, P.B. 1053 Blindern, N-0316 Oslo, Norway

E-mail address: snorrec@math.uio.no 